Review article

\title{
Melanin nanoparticles as a promising tool for biomedical applications - a review
}

\author{
Mariana Caldas $^{\mathrm{a}, \mathrm{b}, \mathrm{c}}$, Ana Cláudia Santos ${ }^{\mathrm{a}, \mathrm{d}, *}$, Francisco Veiga ${ }^{\mathrm{a}, \mathrm{d}}$, Rita Rebelo ${ }^{\mathrm{a}, \mathrm{b}}$, Rui L Reis ${ }^{\mathrm{a}, \mathrm{c}, \mathrm{e}}$, \\ Vitor M Correlo b,c,e \\ a Department of Pharmaceutical Technology, Faculty of Pharmacy, University of Coimbra, Coimbra, Portugal \\ b 3 B's Research Group - Biomaterials, Biodegradables and Biomimetics, Universidade do Minho, Headquarters of the European Institute of Excellence on \\ Tissue Engineering and Regenerative Medicine, AvePark - Parque de Ciência e Tecnologia, Zona Industrial da Gandra, 4805-017 Barco/Guimarães, Portugal \\ ' ICVS/3B's - PT Government Associated Laboratory, Braga/Guimarães, Portugal \\ ${ }^{\mathrm{d}}$ REQUIMTE/LAQV, Group of Pharmaceutical Technology, Faculty of Pharmacy, University of Coimbra, Coimbra, Portugal \\ e The Discoveries Centre for Regenerative and Precision Medicine, Headquarters at University of Minho, Avepark, 4805-017 Barco, Guimarães, Portugal
}

\section{A R T I C L E I N F O}

\section{Article history:}

Received 1 November 2019

Revised 23 January 2020

Accepted 28 January 2020

Available online 1 February 2020

\section{Keywords:}

Melanin

Nanoparticle

Drug delivery system

Theranostics

Imaging

Dioengineering

\begin{abstract}
A B S T R A C T
Melanin is a biopolymer of easy and cheap availability that can be found among the living organisms and excels for its biocompatibility and biodegradability properties, along with scavenging abilities, metal chelation and electronic conductance. This biomaterial can act as a nanocarrier or agent itself to be used in diverse biomedical applications, such as imaging, controlled drug release, bioengineering and bioelectronics, antioxidant applications and theranostics.

In this review, the melanin source and structure, its physicochemical properties, melanin-like polymers as well as the differences among those will be elucidated. The focus will be the discussion of the current approaches that apply melanin nanoparticles (MNPs) and melanin-like nanoparticles (MLNPs) in the biomedical field, to which promising capabilities have been attributed, regarding optoelectronic, photoconductivity and photoacoustic. The use of these nanoparticles, in the last 10 years, in topics as drug delivery or theranostics will be detailed and the major achievements will be discussed. Overall, we anticipate that melanin can drive us toward a new paradigm in medical diagnostics and treatments, since applying melanin features possibly its use as a theranostics nanocarrier agent, not only for diagnostics, but also for photothermal therapy and controlled drug release through chemotherapy.
\end{abstract}

\section{Statement of significance}

We present here a timely and opportune review article focusing the significant potential of melanin nanoparticles in biomedical applications, which will be discussed thoroughly. This biomaterial presents multiple capabilities that may be taken into consideration towards cancer theranostics, expecting a high future impact in the nanosized-platforms design and performance.

(c) 2020 Acta Materialia Inc. Published by Elsevier Ltd. All rights reserved.

\section{Introduction}

Recent therapeutics have evolved regarding specificity and safety improvements, towards the obtainment of more effective treatments over the conventional counterparts. In this context,

\footnotetext{
* Corresponding author. Ana Cláudia Santos, Ph.D, Assistant Professor. Department of Pharmaceutical Technology Faculty of Pharmacy, University of Coimbra (FFUC) Pólo das Ciências da Saúde, Azinhaga de Santa Comba, 3000-548 Coimbra, Portugal.
}

E-mail address: acsantos@ff.uc.pt (A.C. Santos). nanotechnology has been emerging as a promising solution for the development of new delivery systems of contrast agents or drugs, towards specific targets [1]. Due to their particular size, nanoparticles (NPs) present higher selectivity and specificity to a defined target, along with an increased surface area, capable of surface modification, when compared to larger particles, like microparticles [2]. Through surface modification is possible to add specific molecules in order to target cells, such as, tumor tissue or bacteria. NPs are also able to improve the drug loading capacity and to promote a controlled drug delivery. The size of NPs is a distinguished 


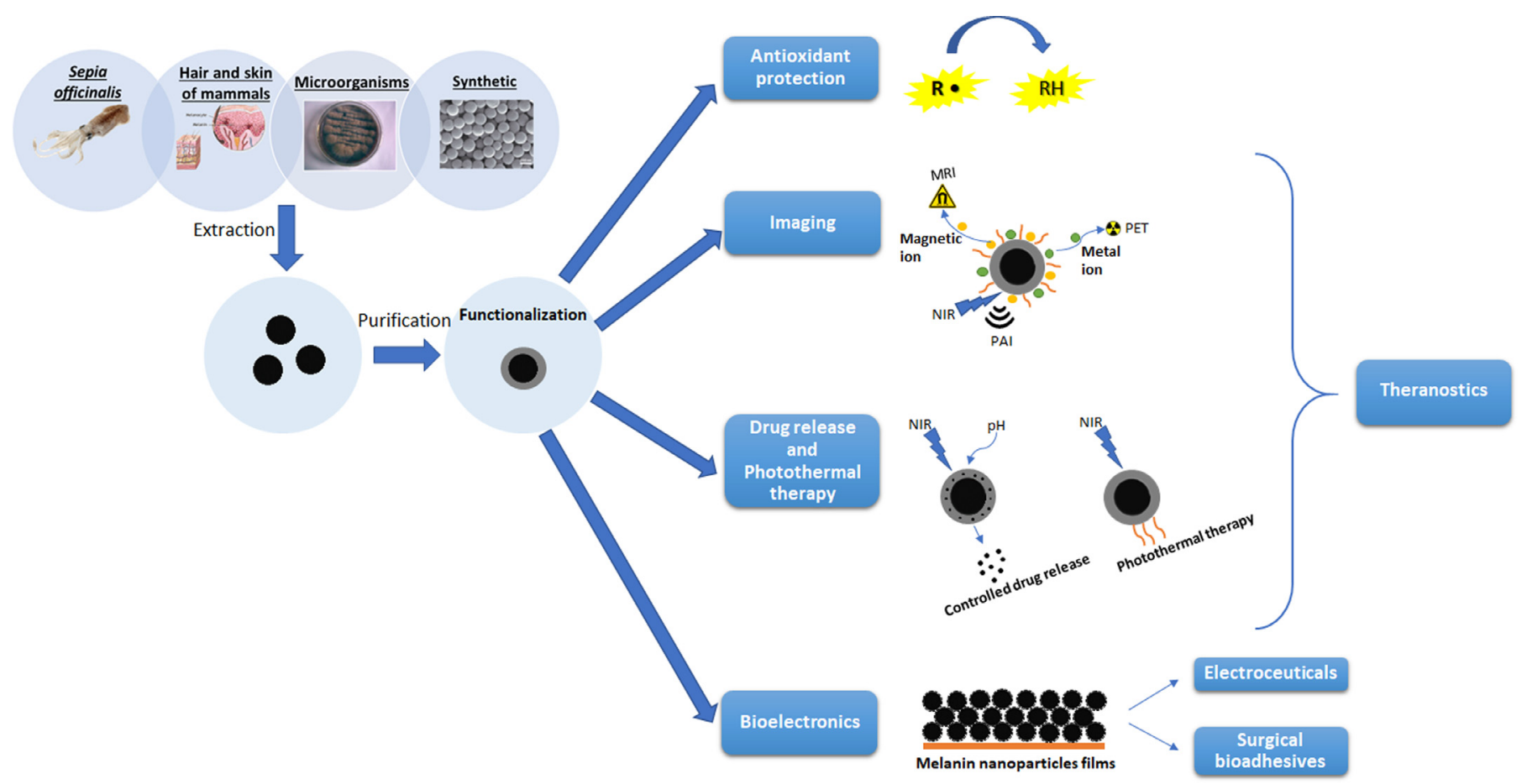

Fig. 1. Schematics of melanin nanoparticles (MNPs) obtainment and biomedical applications.

advantage for the targeting purposes, since, once in the vascular system, these nanosized structures are able to penetrate into cells by endocytosis or receptor-mediated transcytosis, which increases the delivery of the molecules into the cells. Specifically, in cancer cells, the abnormal vessels formed by tumor's angiogenesis are more permeable and the lymphatic drainage is diminished, whose factors increase the concentration of the NPs into tumor cells. This phenomenon is known as the enhanced permeability and retention (EPR) effect [3]. Additionally to these benefits, NPs are able to be excreted through the common organs, such as liver and kidney, more easily than higher-sized particles, showing robust biocompatibility and, in some cases, biodegradability properties. These properties make NPs an outstanding technological strategy for the diverse biomedical areas, such as diagnosis, therapy and also their combinations, the theranostics [4,5].

Despite all the biological processes of human body, the specification of targets regarding drug delivery is extremely important, aiming to target cells without damaging the healthy surroundings, offering a superior efficacy [6]. Several physiological factors are able to induce the controlled release of molecules from delivery systems. For instance, differences in $\mathrm{pH}$ can trigger the release of a molecule at different sites, e.g., the acidotic $\mathrm{pH}$ values of cancer cells may promote the molecule release inside those cells $[7,8]$. Another example is the use of stimuli-sensitive carriers to of ultraviolet (UV) radiations, electrical conductance or magnetic stimulus, promoting the molecules permeation and release in a precise target $[9,10]$.

The search for green biomaterials, which can be easily obtained from the nature, has been growing in the research community. Melanin, a biopolymer found in living organisms and plants, has shown to be very promising among distinct research areas, as biomedicine, dermocosmetics, nanotechnology and bioengineering $[21,39]$. Its biodegradability and biocompatibility assume a strong relevance in the production of biomaterials, owing to the need for biomaterials with no cytotoxicity or side effects. In fact, antigenic response is one of the main difficulties usually associated with the current used biomaterials, along with the lack of stability under physiological conditions. In the case of melanin, this problem is surpassed due to the absence of specific enzymes, in the human body, capable of degrading it, exhibiting additionally valuable attributes of biostability $[11,12]$. On the other hand, melanin in the form of nanoparticles can be excreted through liver and kidney pathways, reducing the possibility of long-term accumulation in organs, usually associated with toxic effects $[13,14]$.

The remarkable features of melanin render it a versatile biomaterial to use in biomedical applications, like controlled drug release, imaging, bioengineering and bioelectronics (Fig. 1). Furthermore, this biomaterial presents a broad absorption of UV-visible radiations, free radical scavenging and metal chelation abilities, together with electronic conductance, antioxidant activity and the capability to bond with different molecules of interest [11,15-18].

This way, due to its intrinsic properties, melanin nanoparticles (MNPs), plays an important role in the development of new controlled delivery systems in the search for new therapies based on the alteration of internal or external conditions.

This review highlights the most recent developments in biomedical field, using natural melanin and melanin-like polymers. Thus, firstly, the structure and source of melanin, and its physicochemical features will be discussed. Secondly, it will be thoroughly analyzed the ongoing research and the future perspectives for this biomaterial, emphasizing several biomedical applications, like magnetic imaging, antioxidant therapy, controlled drug and theranostics, which may benefit from all the potential of MNPs features.

\section{Melanin}

\subsection{Source and structure}

Melanin is a naturally occurring biopolymer present among the living organisms, particularly in the skin and hair of mammals, in the ink of Sepia melanin from Sepia officinalis, in bacteria and additional species. It consists of an heterogeneous molecule, that exists as dark eumelanin or yellow pheomelanin forms, regarding, respectively, the absence or the presence of a sulfur group (Fig. 2). Eumelanin is deserving of greater attention in compari- 


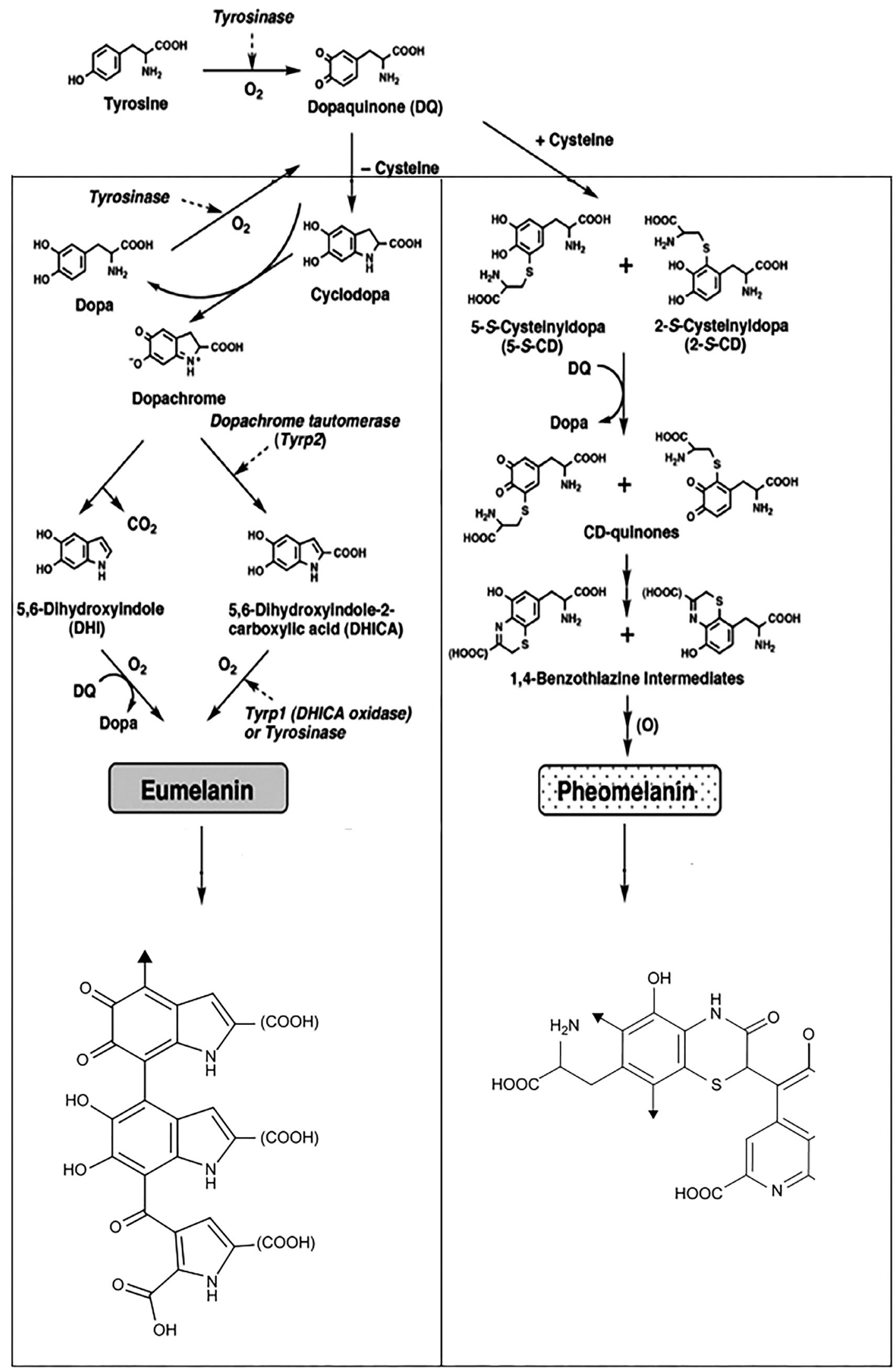

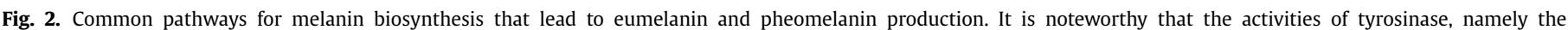

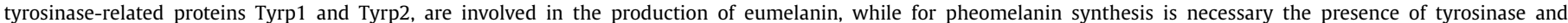
cysteine. Reproduced with permission from [21]. 
son to pheomelanin, since it is more stable and evidences useful characteristics in biomedical applications, whilst pheomelanin evidences lack of photostability and may promote carcinogenesis [19].

Melanin is a very low soluble polymer in water, with the molecular formula $\mathrm{C}_{18} \mathrm{H}_{10} \mathrm{~N}_{2} \mathrm{O}_{4}$, and molecular mass of 318.288 g.mol ${ }^{-1}$ [19]. This molecule structure is controversial among scientific community, since it suffers an extensive biosynthesis process, with several precursors, which can give rise to different macromolecules. These different precursors are biomolecules formed in vivo from different cathecol molecules, by means of the tyrosinase enzyme that turns L-tyrosine into an intermediate, L-3,4-dihydroxyphenylalanine (L-DOPA), and also by a cascade of additional reactions that will result into an oligomer of 5,6-dihydroxyindole (DHI) and its 5,6-dihydroxyindole 2-carboxylic acid (DHICA) form. Depending on the ratio between these two molecules (DHI and DHICA), the physicochemical properties of melanin will change, leading to different final molecule oxidation states, affecting the melanin metal-chelating features, as well as the morphology $[11,15,20]$. The structure of this catechol-derived molecule allows the reaction with additional several molecules, particularly with the ones holding nucleophilic amine and thiol groups, giving origin to protomolecules, which can be further used in different biomedical fields.

Although the primary structure of melanin (composed of DHI and DHICA) is consensual among the scientific community, its macromolecular final structure is still not clear. In fact, depending on the monomers coupling site it can lead to a large heteropolymer or, according to other authors, to a stacked oligomer. Nevertheless, both structural forms lead to the formation of melanin particles with the same chemical properties [15]. More recently, a new model has been proposed for these particles formation, consisting of the building up of a molecule up to its final complex state, through aggregation steps. According to this model, the oligomer sheets of DHI and DHICA form a protoparticle $\left(10^{-9} \mathrm{~m}\right)$ that densifies into a larger protoparticle, creating an onion-like structure, a type-A particle $\left(10^{-8} \mathrm{~m}\right)$, which, in turn, consolidates and agglomerates into a larger and spherical type-B particle $\left(10^{-7} \mathrm{~m}\right)$. This chemical final form of melanin corresponds to the one found in the natural sources, after isolation and purification processes [20].

\subsection{Physicochemical properties}

Establishing the relationship between materials' properties and structure, as well as to understand how this relationship defines the biological behavior of the material, constitutes a great goal among biomedical researchers. Such assumption is observed regarding melanin research, where the aim consists of understanding how its molecular structure influences its macroscopically properties and functions.

In fact, melanin exerts several relevant functions in biosystems, including broad UV and visible absorption band and consequent photoprotective effect, free-radical scavenging and antioxidation, metal chelation, electrical and photoconductivity, as well as some involvement in nerve systems. These functions are defined by its chemical and physical properties, namely the molecular, supramolecular and aggregate-level structures [15,22]. A summary of the physicochemical properties of melanin is presented in Table 1.

It was observed that melanin presents a highly negative surface revealed by zeta potential values which confers stability to the particles by electrostatic repulsion, avoiding aggregation [29]. Melanin appears as a black solid and is almost insoluble in both aqueous and organic solvents [30]. Two of melanin's key functions are coloration and photoprotection. Thus, the optical properties of the melanin are essential to its function. Regarding UV and visible absorption spectrum, melanin evidences a broad-band mono-

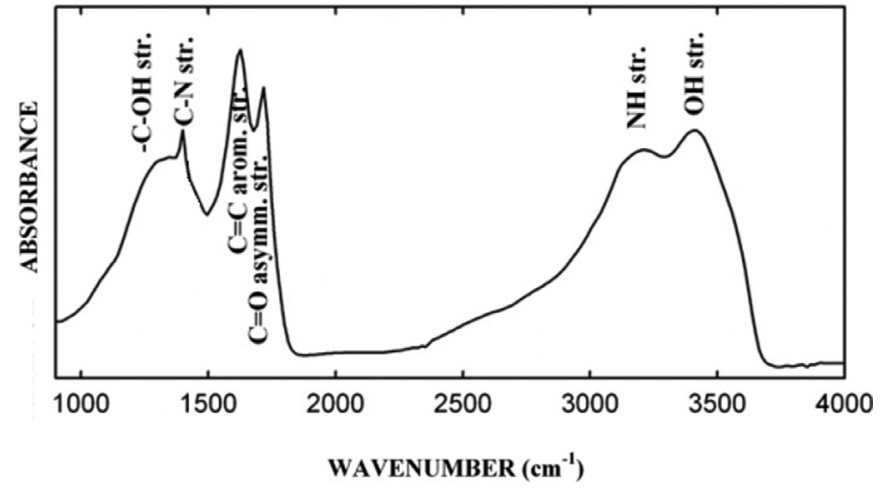

Fig. 3. The fourier-transform infrared spectroscopy (FTIR) spectrum of melanin. Reproduced with permission from [34].

tonic absorption and, interestingly, the spectrum resembles more an inorganic semiconductor material than an organic typical chromophore. In addition, the spectrum is not constant during the formation of the protoparticles. In fact, it evolves during the oxidation and agglomeration of the melanin precursor molecules, associated to the formation of new cross-links $[11,15]$. The fourier-transform infrared spectroscopy (FTIR) spectrum of melanin displays a peak at $3200 \mathrm{~cm}^{-1}$ and $3400 \mathrm{~cm}^{-1}$ due to the $\mathrm{N}-\mathrm{H}$ and $\mathrm{O}-\mathrm{H}$ stretching vibrations of amino and hydroxyl groups, respectively. Aromatic bendings of $\mathrm{C}-\mathrm{C}$ and $\mathrm{C}-\mathrm{N}$ that origin strong peaks at $1600 \mathrm{~cm}^{-1}$ and $1350 \mathrm{~cm}^{-1}$, respectively, plus the carbonyl stretching $\mathrm{C}-\mathrm{O}$ at $1300 \mathrm{~cm}^{-1}$ indicate the aromatic biopolymer nature of melanin by the strong $\pi-\pi$ interactions. At $1300-1100 \mathrm{~cm}^{-1}$ the peaks refer to the presence of phenolic hydroxyl and carboxyl groups, and at $800-600 \mathrm{~cm}^{-1}$ the weak peaks correspond to the substitution of aromatic rings by aromatic hydrogens [31-34]. Fig. 3 displays a FTIR absorbance spectrum of the mentioned peaks with the respective functional groups.

Additionally, melanin holds the ability to absorb the UV light (the absorption increases exponentially towards shorter wavelengths (300-400 $\mathrm{nm}$ ) and converts the absorbed radiation into heat [35]. This photodynamic feature is due to electron-transfer processes, which are conducted by different photoreactions, including anaerobic reactions that occur at wavelengths superior to $300 \mathrm{~nm}$, and aerobic reactions, carried out at shorter wavelengths. The first referred reactions take place only when no external donor or acceptors of electrons exist [15]. The ability to absorb radiation will quench and scavenge excited molecules, promoting a photoprotective effect in melanin pigments. This characteristic is reflected in the protection of human skin by the melanocytes against UV damage [36]. The same protective effect may occur by the melanin extracted from grapes (Vitis vinifera) by the inhibition of the UV-induced lipid peroxidation [37].

Another important feature is the radical scavenging and antioxidant activity that are more related to the existence of DHICA monomers, by transference of $\mathrm{H}$-atoms. This feature is attributed to the chemical structure of the monomers, where the DHICA is less aggregated and stabilized, being more susceptible to react with reactive oxygen species (ROS), which are generated in certain physiological reactions, contrary to the more stable structure of DHI [38].

Additionally, melanin shows strong electrical and photo conductivity, making it a remarkable bio-organic material with promising applications in bioelectronics. The indolic structure of melanin and the electronic delocalization are thought to be responsible for its electrical conductive properties under specific conditions of humidity, temperature and electrical fields. On the other hand, photoconductivity, which under UV or visible light absorption decreases the resistance, is influenced by the oxidation of hydroxyquinones 
Table 1

Physicochemical properties of melanin.

\begin{tabular}{lll}
\hline Properties & Melanin & References \\
\hline CAS registry number & $8049-97-6$ & {$[23]$} \\
Chemical formula & $\mathrm{C}_{18} \mathrm{H}_{10} \mathrm{~N}_{2} \mathrm{O}_{4}$ & {$[24]$} \\
Appearance & Black solid (after purification from S. officinalis) & {$[15]$} \\
Zeta potential & $-43.13 \pm 0.86 \mathrm{Mv}-37.00 \pm 0.10 \mathrm{mV}$ & {$[25,26]$} \\
Molecular weight & $318.288 \mathrm{~g}^{-1} \mathrm{~mol}^{-1}$ & {$[23,27]$} \\
Water solubility & Low soluble $3.397 \mathrm{e}+005 \mathrm{mg} / \mathrm{L} @ 25{ }^{\circ} \mathrm{C}$ & {$[25,28]$} \\
\hline
\end{tabular}

to semiquinones, releasing mobile protons [21]. These properties are owed to the free radicals of the quinones oxidation, as mentioned before, that can reduce or oxidize metals, such as ferric ion $\left(\mathrm{Fe}^{3+}\right)$, cupric ion $\left(\mathrm{Cu}^{2+}\right)$, gadolinium ion $\left(\mathrm{Gd}^{3+}\right)$ or lead ion $\left(\mathrm{Pb}^{2+}\right)$ [39-41].

Finally, melanin can also be involved in the nerve system protection. For instance, the melanin present in the brain (neuromelanin), specifically in the substance nigra, helps neurons to manage the toxic presence of iron and other metals, through metal induced oxidative stress, promoting neuronal survival. This melanin plays a protective role in skin as neuromelanin plays against several harmful factors to neurons $[42,43]$.

\subsection{Natural versus synthetic melanin}

Melanin can be naturally obtained, as outlined in the previous Section 2.1, or synthesized using different approaches in order to produce melanin-like (ML) polymers, from which spontaneously melanin-like nanoparticles (MLNPs) can be obtained, leading to synthetic melanin. The most common approaches make use of dopamine (Da), a catechol molecule with a molecular structure similar to melanin, which, when oxidized, turns into Da-quinone and DHI units, without the presence of DHICA. Da instantly polymerizes into polydopamine (PDa) under solutions containing (tris(hydroxymethyl)aminomethane) (Tris) base or sodium hydroxide, among others basic catalysts, resulting in black NPs, similar to natural melanin granules. The method most similar to the in vivo process of melanin synthesis is by using tyrosinase, in which the product obtained shows to have the most similarity to natural melanin regarding its molecular structure. This approach results in NPs with an average size of $200 \mathrm{~nm}$, similar to those obtained from Sepia officinalis melanin [44].

However, the formed PDa is not exactly the same as the melanin projected models, since the first consists of aggregates of monomers through $\pi-\pi$ and hydrogen bonds, while the second pertains to covalent bonded oligomers. [11,20] Despite the molecular differences, between the NPs obtained from Da and melanin, Da has been receiving higher interest due to the easy synthesis of NPs and the ability to polymerize in situ, which allows the formation of coatings around the NPs.

Due to the presence of catechol and amino groups in PDa it can couple with amine and thiol groups of biomolecules, through nucleophilic bonds, by controlling the pH [45]. Therefore, PDa can be used for several biomedical applications since it has the ability to adhere to different surfaces, such as metals, organic polymers or ceramics.

Regarding the optoelectrical properties, melanin tends to hold a high electret effect, which means storing electrical charges and polarization, depending on the water content. In this context, natural melanin shows a superior water content than the synthetic variants of melanin, as PDa NPs [46].

However, natural melanin can be obtained from numerous sources which will inherently change its physicochemical properties. For example, the distribution of metals in melanin can demonstrate this heterogenicity, such as the presence of $\mathrm{Ca}, \mathrm{Mg}$,
$\mathrm{Na}$, and $\mathrm{K}$ in Sepia melanin, which are generally the most abundant metals in sea water, its natural source. Different studies report changes in the total metal content such as the content determined by Sarzanini et al. [47] is four times greater than the content determined by Liu et al. [48]. This traduces the differences in processing the samples in terms of isolation and preparation of melanin and the need to achieve a set of standardized procedures to obtain homogeneous samples that preserve the inner properties of melanin $[49,50]$.

\section{Biomedical applications}

As discussed previously, melanin evidences remarkable antioxidant, optoelectronic, photoacoustic and photoconductivity properties. Herein, an update comprehensive review is presented by reports that make proof of melanin as a promising green agent capable of improving the diversity of applications in the biomedical field. Table 2 shows some examples and outputs of MNPS in distinct biomedical applications.

\subsection{Antioxidant activity and photoprotective effect}

There has been a growing interest for molecules that gather antioxidant features and can treat specific diseases related with the increment of ROS and oxidative stress. MNPs may offer a great promise towards this need attending due to its radical scavenging properties. Current research has been showing the antioxidant properties of melanin, as a result of its ability to quench excited molecules like dyes, scavenging free radicals and to bind strongly to metal ions $[63,64]$. Ju et al. [51] produced MLNPs, by Da hydrochloride oxidation, with an average size of $80 \mathrm{~nm}$. These MLNPs have persistent electron spin resonance (ESR), which indicates the stability of free radical centers in the material. However, the produced MLNPs have precipitated in PBS (phosphate buffer solution) and FBS (fetal bovine serum) solutions, so, to overcome this drawback, thiol-terminated methoxy-poly (ethylene glycol) (mPEG-SH) was added to the MLNPs surface, conferring a stability up to 2 weeks in the same media. In HeLa cells, the viability of the formulation was studied and, with or without surface modification, the viability was always up to $85 \%$, which suggested a good biocompatibility even after the surface modification. The radical scavenging property of the MLNPs was studied by an 2,2-diphenyl-1-picrylhydrazyl (DPPH) assay and it was evaluated by electrophoretic light scattering (ELS). The results exhibited an increase on the radical scavenging as the concentration increased to 85 and 79\%, respectively, for MLNPs and mPEG-SH-MLNPs. These results were also confirmed by ESR, where the signal from the free radical DPPH decreased as the MLNPs concentration increased. Finally, as the size of the MLNPs were smaller than Sepia officinalis eumelanin, the efficient concentration $\left(\mathrm{EC}_{50}\right)$ needed to decrease to $50 \%$ the initial amount of DPPH was diminished, which could be explained by the increase in surface area that lead to a superior scavenging activity. These values were very similar with ascorbic acid, a commonly used free radical scavenging material. Although this work doesn't give much insight into possible clinical 
Table 2

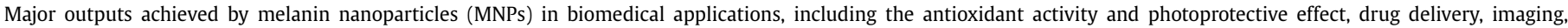
theranostics, and bioengineering and bioelectronics.

\begin{tabular}{|c|c|c|c|c|c|}
\hline Applications & Source & Methodology of production & Particle size $(\mathrm{nm})$ & Major outputs & Ref. \\
\hline \multirow[t]{5}{*}{$\begin{array}{l}\text { Antioxidant Activity and } \\
\text { Photoprotective effect }\end{array}$} & $\begin{array}{l}\text { Dopamine } \\
\text { hydrochloride }\end{array}$ & Oxidation & $<100$ & $\begin{array}{l}\text { - Good biocompatibility with HeLa cells } \\
\text { - Free radical scavenging activity }\end{array}$ & [51] \\
\hline & $\begin{array}{l}\text { The ink of Sepia } \\
\text { officinalis }\end{array}$ & Washing and purification & $50-300$ & $\begin{array}{l}\text { - Internalization by primary human keratinocytes } \\
\text { (hKCS) } \\
\text { - Reduced amount of reactive oxygen species } \\
\text { (ROS)/reactive nitrogen species (RNS) released by } \\
\text { UVA/UVB irradiated hKCs }\end{array}$ & [40] \\
\hline & Synthetic & Sol-gel & $<80$ & $\begin{array}{l}\text { - Prevention of the typical tendency to aggregate and } \\
\text { uncontrolled particle growth } \\
\text { - Efficient cytoprotective effects }\end{array}$ & [52] \\
\hline & & Enzymatic polymerization & $<70$ & - Protection of bone marrow from ionizing radiation & [30] \\
\hline & & Oxidation & 80 & - Effective radioprotection against $\gamma$-radiation & [29] \\
\hline \multirow[t]{4}{*}{ Drug delivery } & $\begin{array}{c}\text { Lactosylated } \\
\text { dopamine }\end{array}$ & Oxidative polymerization & $<150$ & $\begin{array}{l}\text { - Strong near infrared (NIR) light absorbance } \\
\text { - Drug-loading capacity of } 66-73 \text { wt\% } \\
\text { - Tunable active-targeting effect } \\
\text { - Synergistic effect of chemo-photothermal therapy } \\
\text { for the ablation of cancer cells }\end{array}$ & [53] \\
\hline & $\begin{array}{l}\text { Dopamine } \\
\text { hydrochloride }\end{array}$ & $\begin{array}{l}\text { Oxidation and } \\
\text { self-polymerization }\end{array}$ & $<100$ & $\begin{array}{l}\text { - Loading capability of distinct drugs and protection } \\
\text { from unresponsive leakage in physiological conditions } \\
\text { - Drug-retaining capacity } \\
\text { - Multi-responsive drug controlled release } \\
\text { - Good stability } \\
\text { - Photothermal effect }\end{array}$ & [54] \\
\hline & & & $\approx 160$ & $\begin{array}{l}\text { - Widely biodistribution throughout the human body } \\
\text { - Strong NIR absorption and high photothermal } \\
\text { conversion efficiency }\end{array}$ & [55] \\
\hline & $\begin{array}{l}\text { The ink of Sepia } \\
\text { officinalis }\end{array}$ & Washing and purification & $100-250$ & $\begin{array}{l}\text { - Promising antibiotic nanodelivery system } \\
\text { - Possibility of diverse bioactive molecules loading by } \\
\text { functionalized nanoparticles }\end{array}$ & {$[25]$} \\
\hline \multirow[t]{2}{*}{ Imaging } & Synthetic & Vigorous stirring & $<10$ & $\begin{array}{l}\text { - Good in vivo tumor imaging properties } \\
\text { - Magnetic resonance imaging (MRI) contrast agent } \\
\text { for tumor-targeted imaging } \\
\text { - Higher relaxivity than that of commercial } \\
\text { - GadodiamideGood tumor-targeting specificity }\end{array}$ & $\begin{array}{l}{[56]} \\
{[13]}\end{array}$ \\
\hline & $\begin{array}{l}\text { Dopamine } \\
\text { hydrochloride }\end{array}$ & $\begin{array}{l}\text { Oxidation and } \\
\text { self-polymerization }\end{array}$ & $<50$ & $\begin{array}{l}\text { - Contrast agent for optoacoustic tomography } \\
\text { - Good dispersion stability of high concentration of } \\
\text { melanin nanoparticles (MNPs) in biological media } \\
\text { - Lower cell toxicity than that of gold nanorods (for } \\
\text { concentrations that provide equal optical absorbance) }\end{array}$ & {$[12]$} \\
\hline \multirow[t]{6}{*}{ Theranostics } & Black sesame seeds & Washing and purification & $20-200$ & $\begin{array}{l}\text { - Absence of long-term toxicity in mice } \\
\text { - Photothermal effect, with significant tumor growth } \\
\text { suppression }\end{array}$ & [31] \\
\hline & $\begin{array}{l}\text { The ink of Sepia } \\
\text { officinalis }\end{array}$ & Washing and purification & $<200$ & $\begin{array}{l}\text { - Strong absorption at NIR region, and higher } \\
\text { photothermal conversion efficiency } \\
\text { - Blood retention and effective accumulation at tumor } \\
\text { sites }\end{array}$ & [32] \\
\hline & $\begin{array}{l}\text { Dopamine } \\
\text { hydrochloride }\end{array}$ & $\begin{array}{l}\text { Oxidation and } \\
\text { self-polymerization }\end{array}$ & $<200$ & $\begin{array}{l}\text { - Optical absorption in the NIR region, which greatly } \\
\text { enhanced the photothermal therapy treatment } \\
\text { efficiency and photoacoustic contrast ability } \\
\text { - Therapeutic efficiency }\end{array}$ & [57] \\
\hline & & & 120 & $\begin{array}{l}\text { - Photoacoustic imaging agent } \\
\text { - Highly efficient photothermal therapy agent }\end{array}$ & [58] \\
\hline & & & $<200$ & - MRI contrast improvement & [59] \\
\hline & Synthetic & $\begin{array}{l}\text { Vigorous stirring } \\
\text { Co-precipitation }\end{array}$ & $\begin{array}{l}<10 \\
<150\end{array}$ & $\begin{array}{l}\text { - Promising for multimodality-imaging guided therapy } \\
\text { - Good photoacoustic signal intensity } \\
\text { - Effective NIR absorbance } \\
\text { - Effective photothermolysis treatment }\end{array}$ & $\begin{array}{l}{[60]} \\
{[33]}\end{array}$ \\
\hline \multirow[t]{3}{*}{$\begin{array}{l}\text { Bioengineering and } \\
\text { Bioelectronics }\end{array}$} & $\begin{array}{l}\text { The ink of Sepia } \\
\text { officinalis }\end{array}$ & $\begin{array}{l}\text { Washing and } \\
\text { purificationLayer by } \\
\text { Layer }\end{array}$ & $<200$ & $\begin{array}{l}\text { - Durable electrochemical conductivities } \\
\text { - Reduced secretion of inflammatory cytokines }\end{array}$ & [26] \\
\hline & $\begin{array}{l}\text { Dopamine } \\
\text { hydrochloride }\end{array}$ & $\begin{array}{l}\text { Oxidation and } \\
\text { self-polymerization }\end{array}$ & $<200$ & $\begin{array}{l}\text { - Antimicrobial properties (in vitro) } \\
\text { - Development of MNPs-based adhesive interfaces for } \\
\text { surgical membranes with remarked capabilities of } \\
\text { bioadhesion }\end{array}$ & [61] \\
\hline & Synthetic & Vigorous stirring & - & $\begin{array}{l}\text { - Efficiently transducing proton-to-electron currents } \\
\text { - Operation via so-called volumetric gating, a } \\
\text { pre-requisite for further miniaturization }\end{array}$ & [62] \\
\hline
\end{tabular}


translation, it was an important door to the leading research of the application of this type of material in biomedical fields, creating a great potential for melanin particle-based high-tech materials.

Da Silva et al. [40] considered the use of melanin in skin wound healing and, for that, they extracted MNPs from the ink of Sepia officinalis, with the typical round nano-sized morphology and electrical conductivity of $2.09 \times 10^{-8} \mathrm{~S} . \mathrm{cm}^{-1}$. In this study authors incorporated MNPs into gellan gum spongy-like hydrogels, in order to achieve a controlled and sustained release of MNPs, which would benefit skin healing, through a direct effect over keratinocytes, as it stimulates the propagation of endogenous electrical signals that attract host regenerative cells. For that, and to evaluate the cytotoxicity and antioxidant properties, MNPS were release into human primary keratinocytes cultures, at different amounts. Furthermore, MNPS were incorporated into spongy-like hydrogels to evaluate the release kinetics and to confirm that melanin-containing hydrogels do not elicit a negative host reaction. In the end of their study, authors were able to observe the antioxidant properties, through the reduced amount of ROS released by UVA/UVB irradiated human primary keratinocytes pre-exposed to it. Furthermore, a possible photo protective behavior was observed, through the internalization of eumelanin NPs by primary human keratinocytes and their disposed around the nuclei of the cells as in native skin. This system represents a good and efficient approach to skin wound healing and minimization of tissue necrosis, using the advantages of MNPS and, consequently, its properties.

Using a different approach, MLNPs were produced by a sol-gel methodology, whereas DHICA was conjugated with 3aminopropyltriethoxysilanes (APTS) and further integrated into a silica nanoscaffold matrix, which increased the stability and biocompability of the MLNPs. The cellular uptake of the Silica-MLNPs (Si-MLNPs) was assessed in human ovarian cancer cells and it was proved to be very fast without cytotoxic effects, with the MLNPS persevered into the cells up to 6 days. Furthermore, cytoprotective effects were assessed against hydrogen peroxide $\left(\mathrm{H}_{2} \mathrm{O}_{2}\right)$-induced apoptosis by the incubation of HeLa and A2780 cell lines with Si-MLNPs during $24 \mathrm{~h}$ followed by $30 \mathrm{~min}$ with $\mathrm{H}_{2} \mathrm{O}_{2}$. No observed effects were detected during the $24 \mathrm{~h}$ caused by the MLNPs internalization, demonstrating their biocompability. In fact, a protective effect of the cells was observed by the decrease of cellular apoptosis normally induced by $\mathrm{H}_{2} \mathrm{O}_{2}$, when compared to cells untreated with Si-MLNPs (control). This process may be due to the acceleration of $\mathrm{H}_{2} \mathrm{O}_{2}$ decomposition, promoted by the MLNPs, without damaging the structure of the Si-MLNPs. This methodology brings a new insight into a possible formulation for biomedical applications based in the intrinsic antioxidant feature of the produced Si-MLNPs [52].

Melanin has several aromatic oligomers that contain $\pi$ electrons in its structure, so when a generated electron passes through the pigment it will gradually lose energy until it can be imprisoned into free radicals present in melanin, preventing the formation of free harmful radicals. This controlled dissipation of high-energy can be useful for example in radioimmunotherapy (RIT) and external beam radiation therapy (EBRT), whose radiation is toxic for bone marrow. Using these principles, MLNPs, which can be delivered into the bone marrow and therefore protect hematopoietic cells against RIT and EBRT, were developed, leading to increase the safety of these treatments. MLNPs were produced by Schweitzer et al., using 5-S-cysteinyl-DOPA and LDOPA, and they were labeled with 188Re, so they could be traced in vivo, which up to $24 \mathrm{~h}$ were efficiently stable in mouse serum. Regarding biodistribution, the uptake of MLNPs by the bone marrow was superior when mice were pre-injected with pluronic acid after $3 \mathrm{~h}$, (6\% contrary to $0.3 \%)$. In vivo studies using CD- 1 mice, after injection with MLNPs and gamma irradiation, revealed a protective effect after 3 and 10 days post-irradiation, suggested by the increase of WBCs (white blood cells) and platelets, even at higher amount of radiation, contrary to controls (absence of MLNPs). Comparatively, the same results were observed for tumorbearing mice treated with RIT, even at very high radiation doses. The myelotoxic effects of these kind of therapies, which are known for their high adverse effects, can be partially overcome by using MLNPs, which provide bone marrow protection and the possibility to use high doses of radiation, leading to a safer and more effective treatment [30].

Likewise, in another study, MLNPs were produced using the same approach as Ju et al. [51], by polymerization of Da hydrochloride. To study the photoprotective effects of MLNPs, mice were divided into five different groups: control (injected intraperitoneally (i.p.) with PBS); melanin control (injected i.p. with MLNPs); melanin pre-irradiation (injected i.p. with MLNPs before gamma irradiation); melanin post-irradiation (injected i.p. with MLNPs after gamma irradiation) and radiation control (injected i.p. with PBS and exposed to gamma radiation). Mice treated with MLNPs along with radiation showed an increase in WBC, platelets, hemoglobin ( $\mathrm{Hb}$ ) and RBCs (red blood cells) concentration, contrary to the remaining groups. The decrease of the previous, after the irradiation, is a reflection of the damage caused by the radiation in stem cells located in the bone marrow, which are more sensitive to the damage. Changes in spleen after irradiation are very common, such as hyperplasia, lymphocyte necrosis and decrease of megakaryocytes. Mice treated with MLNPs before irradiation showed less changes in the spleen compared to other groups, along with an increase in megakaryocytes, acting like an immunomodulator. Simultaneously, in order to evaluate the possible damages of DNA, a comet assay was conducted. The values of comet assay, i.e., \%DNA in tail, tail length, tail moment, and olive moment, displayed an increase by $8,4,17$ and 10 folds in the radiation control group compared with control group. On the other hand, the melanin preand post-irradiation groups showed a decrease in the comet parameters over time, compared with the radiation control group, protecting the DNA. The survival rate of MLNPs injected-mice was improved by $30 \%$ compared to only $10 \%$ of the non-injected group. These promising results suggest MLNPs as a viable strategy to promote radioprotection of bone marrow tissue, liver, spleen and other tissues, along with immunomodulator effects when a patient is subjected to radiation or electrical pulses [29].

\subsection{Drug delivery and therapeutic systems}

Melanin has been used as a drug carrier to prepare formulations without further drying or purification steps and no need for solvents' use. [65] Its beneficial properties, namely the fact of being a low soluble biopolymer, together with the spontaneously formation capacity of particles at the nanometric scale, offer great promise to this biomaterial to be exploited in drug delivery systems $[25,66]$.

Photothermal therapy (PTT) has emerged as a minimal invasive approach to kill cancer cells without damaging the healthy tissue around the abnormal cells. This strategy is possible as a result of the absorbance of energy by the MNPs, induced by near infrared (NIR) light, allowing MNPs to release a great amount of vibrational energy, which turns into heat $[67,68]$. The possibility to induce heating by NIR light irradiation, in the specific site where tumor is located, enhances the targeting effect and efficacy of the therapeutics.

As an example, Liu et al. [55] synthesized MLNPs through the oxidation of Da hydrochloride, originating colloidal nanospheres. The particles were stable, without agglomerating signals, and were dispersible in $10 \%$ blood serum solution without any changes, which certified their potential to be used in vivo. The photothermal effect was studied by the application of NIR irradiation, which trig- 
A)

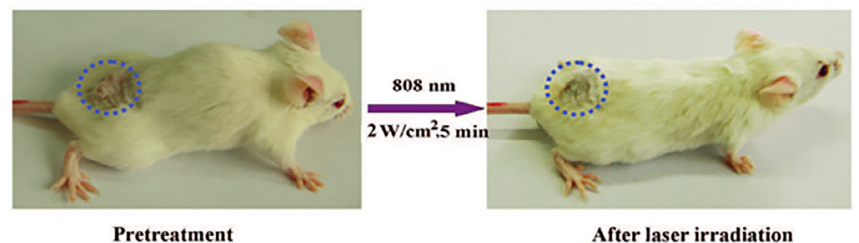

c)

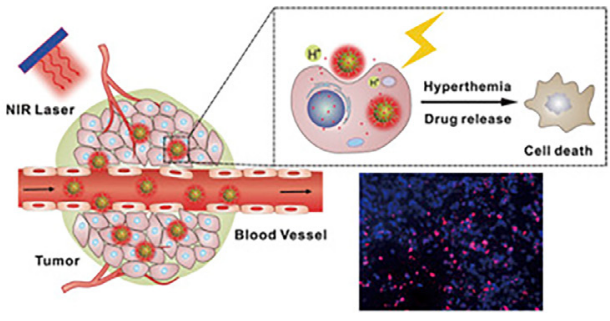

D)
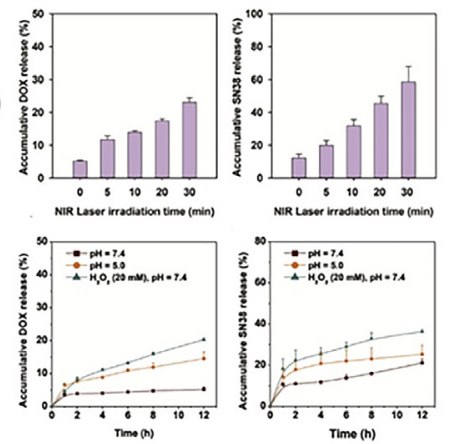

B)

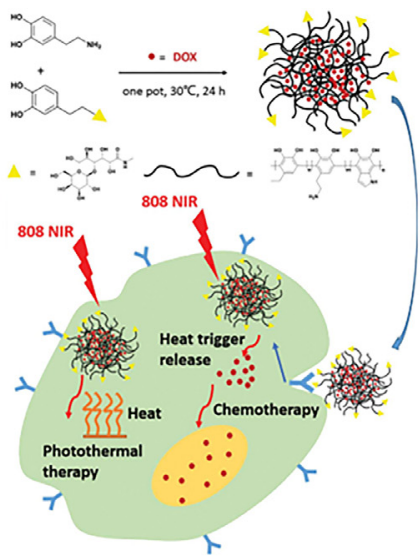

E)

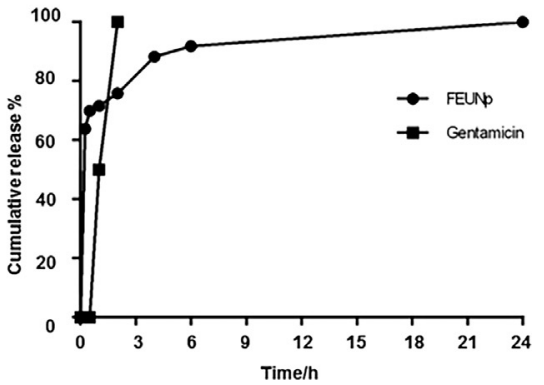

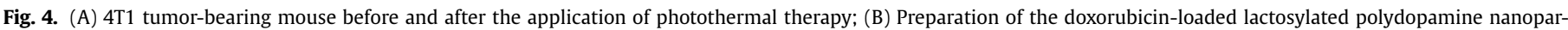

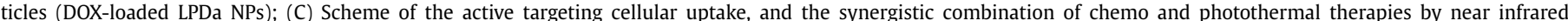

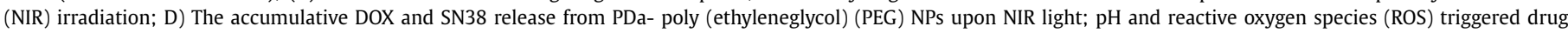

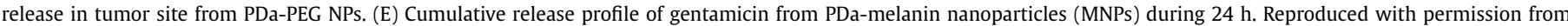
$[25,53-55]$. (For interpretation of the references to colour in this figure legend, the reader is referred to the web version of this article.)

gered a temperature enhancement of $33^{\circ} \mathrm{C}$ (from $25{ }^{\circ} \mathrm{C}$ to $58{ }^{\circ} \mathrm{C}$ ), contrary to water control that exhibited a minor increase of $3{ }^{\circ} \mathrm{C}$ (from $25{ }^{\circ} \mathrm{C}$ to $28{ }^{\circ} \mathrm{C}$ ). In fact, to destroy cancer cells, the temperature should be maintained during $15-60 \mathrm{~min}$ at $43{ }^{\circ} \mathrm{C}$, approximately, or during 5 min over $50{ }^{\circ} \mathrm{C}$ [69]. Using these MLNPs, the photothermal conversion efficiency proved to be much higher (40\%) than for the commonly used gold nanorods. Another important feature is that, after NIR irradiation, MLNPs didn't show any morphology changes, evidencing photostability, contrary to gold nanorods that presented loss of absorbance, due to their melting, triggered by the photothermal effect. In vitro cytotoxicity assays were conducted in 4T1 and HeLa tumor cells, and, following the incubation with MLNPs and NIR irradiation for $5 \mathrm{~min}$, most of the cells were dead, contrary to controls (only NIR or MLNPs), which accounted for $20 \%$ and $90 \%$ of cellular viability, respectively. Afterwards, Balb 4T1 tumor-bearing mice were injected with MLNPs and, after NIR irradiation, almost all of the tumor tissue was necrotic, and no regrowth was observed, dissimilar to the continuous growth of tumor in control groups. The mice, after one month of treatment, showed no signal of adverse effects (Fig. 4A), confirming, once more, the remarkable biosafety of this material in the use of cancer treatment [55].

The presented features of the MLNPs make possible the use of those structures as a chemo-PTT agent against cancer cells, combining the PTT and the most common anti-cancer approach, chemotherapy, leading to a desired synergistic effect. In a study performed by Gao et al. [53], lactosylated PDa (LPDa) was used to produce MLNPS as a nanocarrier for the anticancer drug doxorubicin (DOX) (Fig. 4B). The photothermal effect was studied and a high photothermal conversion efficiency (about 40\% compared to the $10 \%$ conferred by standard gold nanorods) was demonstrated [55]. After proving that LPDa NPs would be a great candidate as a nanocarrier for PTT, DOX was encapsulated into the NPs during the polymerization process. This process resulted in a $66-73 \%$ of drug loading efficiency and a cumulative drug release of $33.4 \%$, reporting to a 1.3-fold enhancement, in comparison to the control (14.6\%). In vitro cytotoxicity assays were conducted using HeLa cells, and tested in two different ways. First, the photothermal effect was tested by incubating the cells and irradiating them with NIR, evidencing a lower cellular viability with the increase in LPDa NPs concentration, obtaining an $\mathrm{IC}_{50}$ around $60 \mu \mathrm{g} \mathrm{mL} \mathrm{m}^{-1}$, confirming their efficient cancer cells killing effect. Next, the DOX-loaded LPDa NPs were incubated in the cell lines and, without radiation, an $\mathrm{IC}_{50}$ around $43 \mu \mathrm{g} \mathrm{mL}-1$ was observed. Finally, to confirm the synergistic effect of the two combined therapies, DOX-loaded LPDa NPs together with the NIR radiation showed an $\mathrm{IC}_{50}$ of ca. $12 \mu \mathrm{g} \mathrm{mL} \mathrm{m}^{-1}$, which was found to be the lowest value compared with the two therapies alone, suggesting a synergistic effect for the combined chemo-photothermal cancer therapy. Even though the drug release from the NPs wasn't very high, the ability to gather two different approaches to directly kill cancer was demonstrated. However, additional studies, such as in vivo studies, should be undergone to sustain the clinical translation of this system.

Comparatively, in a similar study, PDa was used as well, although it was additionally modified with poly (ethylene glycol) (PEG) on the surface. The use of photothermal and chemotherapies was the strategy adopted to destroy cancer cells and diminish the side effects offered by the current used treatments (Fig. 4C). Two anticancer drugs, namely DOX and 7-ethyl-10hydroxycamptothecin (SN38), were used and loaded into the NPs. PEG-modification confirmed to not interfere with the UV-Vis adsorption of Da and, the negative surface charge of PDa-PEG NPs in water (ca. $-23 \mathrm{mV}$ ) and PBS $(-19 \mathrm{mV}$ ) allowed for an easy transport through blood circulation. The photothermal effect was 
assessed at different concentrations of PDa-PEG NPs and a higher effect was observed as the concentration increased. The PDA-PEG NPs are rich of phenyls, amino and hydroxyl that bestows them the ability to bound drugs such as DOX and SN38, via $\pi-\pi$ stacking and hydrogen bond interaction. After drugs loading, the stability and morphology of the PDa-PEG NPs was preserved. Drug release from the PDa-PEG NPs was studied using different stimuli. NIR irradiation was used and the drug release was increased by the time of the irradiation. After $30 \mathrm{~min}$, ca. $23 \%$ of DOX and ca. $59 \%$ of SN38 were released (showing a burst release upon irradiation), contrary to control, where no radiation was used, and which release was ca. $5 \%$ and ca. $12 \%$, respectively. Additionally, the drugs could be released in an acidic condition due to the destruction of the amine groups formed between the drugs and PDA-PEG NPs. Under oxidative conditions, using $\mathrm{H}_{2} \mathrm{O}_{2}$, the polyphenol groups of PDa are oxidized leading to the breakage of the formed hydrogen bonds between PDa and drugs. Consequently, a release of ca. 20\% for DOX and ca. 36\% for SN38, approximately, was achieved after $12 \mathrm{~h}$ of incubation (Fig. 4D). Both findings assume relevant importance, once, in tumor cells, the environment is simultaneously acidic and under oxidative stress, constituting the necessary stimuli to trigger the release of the drugs. Cell viability assays were conducted both in non-malignant NIH-3T3 cells and in malignant MCF-7and PC-9 cells, and PDa-PEG NPs were found to be nontoxic. The photothermal efficacy was enhanced along with the increased concentration of PDA-PEG NPs, evidencing an $\mathrm{IC}_{50}$ around $60 \mathrm{mg} \cdot \mathrm{mL}^{-1}$, which was fixed for the subsequent studies. Furthermore, individual photothermal or chemotherapeutic treatments showed to slightly diminish the cellular viability on cancer cells, but not sufficiently, while the combination of the therapies evidenced significant visible effects towards the decrease of the cell viability, emphasizing the accomplishment of strong synergistic effect. In vivo assays were carried using DOX-loaded PDA-PEG NPs in tumor-bearing mice. No significant differences were observed from the control groups (only NIR, free DOX) and DOX-loaded PDa-PEG group on excised tumors, indicating their inefficacy to suppress the tumor without radiation. The tumor-site temperature was enhanced only in PDa-PEG NIR group and DOX-loaded PDa-PEG NIR group, which was owed to the Da photothermal effect. Moreover, free DOX caused cardiotoxicity and high loss of body weight. Contrarily, on the PDaPEG NIR group, the tumor suppression was more noticeable. However, the efficacy of the treatment was more effective on the DOXloaded PDa-PEG NIR group, sustaining the hypothesis that chemoand photothermal therapies are synergic methodologies with a notorious safety, according to the absence of pathological changes in mice during and post-treatment. This kind of drug nanocarrier constitutes a prominent advantage for personalized medicine, by controlling the treatment in time and to allow for specific drugs dosages, increasing the efficacy and diminishing the side effects of the therapy. [54]

Towards a distinct therapeutic application, MNPs were applied for the delivery of an anti-infectious drug, using them as a nanocarrier for metronidazole, aiming its deliver to the colon and intestines, by a pH-responsive release. In this study, metronidazole was incorporated into the MNPs, through a supercritical $\mathrm{CO}_{2}$ technology, which allows the absence of solvents use, together with additional drying or purification steps. In this process, ca. $2 \% \mathrm{wt}$ of metronidazole was incorporated into the MNPs. Free metronidazole didn't show variations in solubility in the different evaluated $\mathrm{pH}$, namely 2.2 and 7.4, contrary to the metronidazole-loaded MNPs, which showed a pH-triggered release, proving the capacities of the biopolymer as a modulator of drug release. In fact, at $\mathrm{pH} 7.4$, about $87 \%$ of metronidazole was released during $9 \mathrm{~h}$, while at $\mathrm{pH} 2.2$ only $10 \%$ of metronidazole was released. This suggests MNPs as a good candidate for drug release in specific organs, with close to neutral or slightly alkaline $\mathrm{pH}$, such as intestines, avoid- ing the premature release in stomach, where an acidic $\mathrm{pH}$ sustains. This beneficial feature of MNPs may be due to the presence of carboxylic groups that, at neutral or slightly alkaline $\mathrm{pH}$, are negatively charged, triggering the repulsion between them, while promoting the release of the encapsulated drug. In another hand, at acidic $\mathrm{pH}$, the drug is found to be more retained, probably due to the network formed between the carboxylic groups, which are protonated. Moreover, the antibacterial activity was assessed using a Resazurin assay and Escherichia Coli (E. Coli) as the model bacteria of the colon. The adherence and proliferation of cells decreased in the presence of metronidazole, specifically at concentrations around ca. $4 \mathrm{mg} \mathrm{mL}^{-1}$ after $24 \mathrm{~h}$, caused by the release of the drug from the MNPs. This study clearly exclaimed the potentiality of MNPs as a nanocarrier for the specific delivery of drugs in colon and intestines, by means of a pH-responsive release [65].

Still on the delivery of antibiotics, a new method to functionalize NPs, without using solvents and additional complex and inefficient processes, was used to incorporate gentamicin into MNPs. Natural melanin was extracted from the ink of Sepia officinalis and purified after washing processes. To incorporate gentamicin into MNPs surface, PDa was used as the functionalizing agent, as it is an adhesive macromolecule that self-polymerizes in specific conditions. Gentamicin-loaded PDa-MNPs underwent in vitro release tests and, although those suffered an initial drug burst release of $60 \%$, it was followed by a sustained drug release profile for $24 \mathrm{~h}$ (Fig. 4E). Finally, the antimicrobial test against E. coli and Staphylococcus aureus indicated that the formulation was effective against the bacteria, revealing a $\mathrm{IC}_{50}$ : ca. $54 \mu \mathrm{g} \cdot \mathrm{mL}^{-1}$ and ca. $110 \mu \mathrm{g} \cdot \mathrm{mL}^{-1}$, respectively. These results confirm the success of MNPs as a promising nanocarrier for the delivery of antimicrobial drugs, which are capable of reducing the inherent toxicity of the encapsulated drug, while attributing its recognized radical scavenger properties [25].

\subsection{Imaging}

Multimodal imaging involves different strategies to upgrade the clinical diagnostics and improve therapeutic results. The greatest challenge is to obtain a biocompatible carrier that is capable to load different contrast agents and to maintain a small size while assuring targeting ability. Some biomolecules based nanocarriers, such as liposomes, have been used, however those do not exhibit sufficient contrast properties to be used in the imaging field $[70,71]$.

Melanin, as already referred, evidences not only strong light absorbing but also magnetic and electrical features that, once together, can project this molecule as a new functional biomarker for multimodality imaging purposes.

Regarding these promising optoelectrical, photoacoustic and photoconductivity properties, researchers [56] extracted melanin from melanocytes to obtain water soluble MNPs. Their surface was modified with PEG and arginylglycylaspartic acid (RGD), a commonly used peptide regarding its tumor targeting features towards the $\alpha \mathrm{v} \beta 3$ integrins of cancer cells. Afterwards, ${ }^{64} \mathrm{Cu}^{2+}$ and $\mathrm{Fe}^{3+}$ were also added to the MNPs, individually and simultaneously, in order to specify their synergic application for position emission tomography (PET) and magnetic resonance imaging (MRI), respectively. Hence, different types of MNPs were obtained with the intuit of study their individual and combined properties: ${ }^{64} \mathrm{Cu}^{2+}$ RGD-PEG-MNPs; $\mathrm{Fe}^{3+}$-RGD-PEG-MNPs and ${ }^{64} \mathrm{Cu}^{2+}-\mathrm{Fe}^{3+}$-RGD-PEGMNPs. After incubation with NIH-3T3 and U87MG cell lines all the formulations revealed to be biocompatible with low signs of cytotoxicity. To study the in vivo photoacoustic properties of the RGDPEG-MNPs, they were injected into U87MG tumor bearing mice, triggering a substantial increase of the signal after $4 \mathrm{~h}$. Such result might be due to the targeting ability of RGD and the EPR effect. 
A)

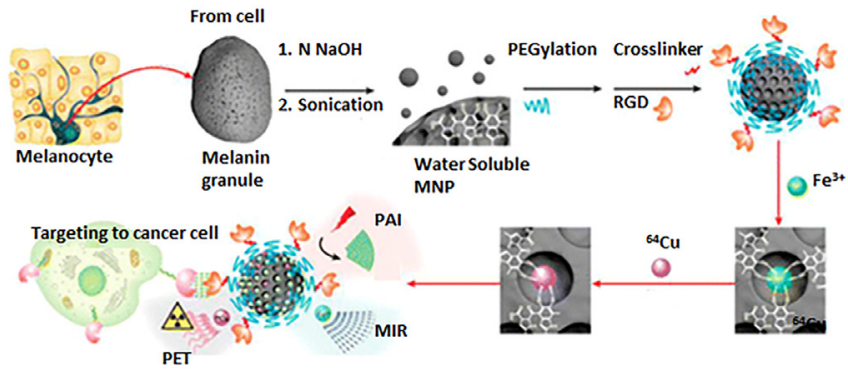

C)

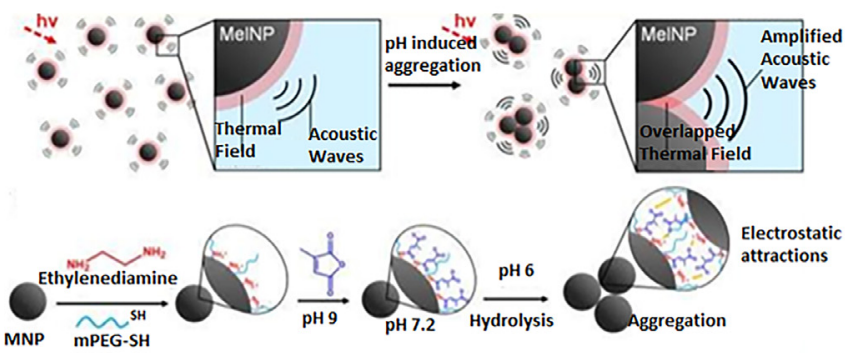

B)

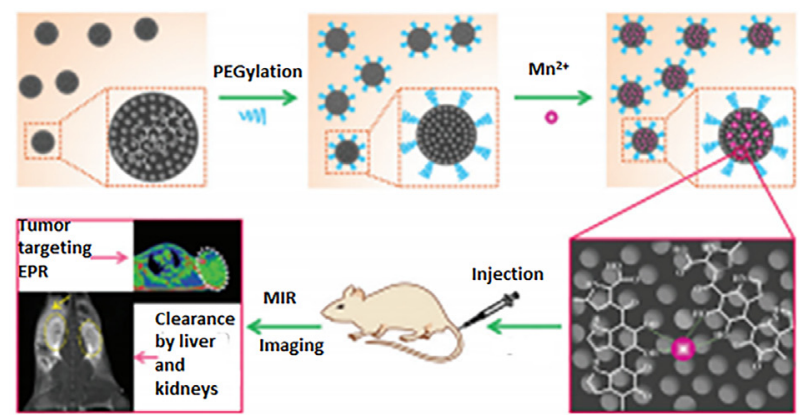

D)

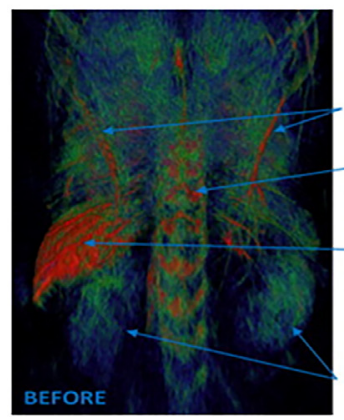

Blood

Vessels

Spine

Spleen

Kidneys

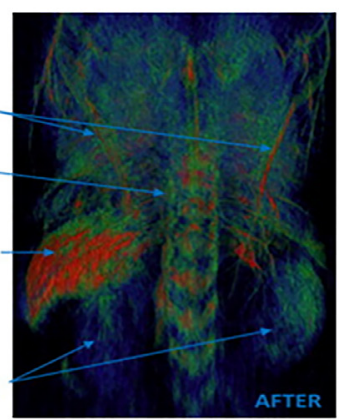

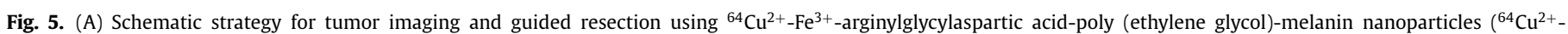

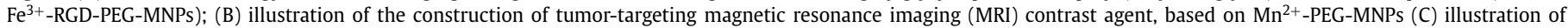

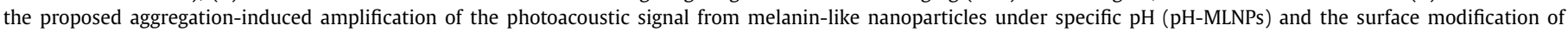

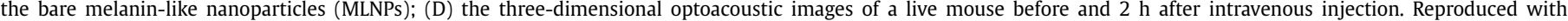
permission from $[12,13,56,73]$.

The magnetic sensitivity of the $\mathrm{Fe}^{3+}$-RGD-PEG-NPs was tested in mice and, after $4 \mathrm{~h}$ of this MNPs subcutaneous injection, the relative signal intensity increased by, approximately, 30\%. Distinctly, the PET imaging features was tested using ${ }^{64} \mathrm{Cu}^{2+}$-RGD-PEG-MNPs, revealing a strong tumor contrast in the tested mice, after $2 \mathrm{~h}$. Finally, the possibility of multimodal imaging was investigated by combining both chelating agents, ${ }^{64} \mathrm{Cu}^{2+}$ and $\mathrm{Fe}^{3+}$, being the results very similar to those observed individually. An important feature of the metals used is that ${ }^{64} \mathrm{Cu}^{2+}$ ions used in PET decay to zinc ions $\left(\mathrm{Zn}^{2+}\right)$ which together with $\mathrm{Fe}^{3+}$ are necessary in the living body, making them less toxic than the regular metals used for imaging. Such positive observed results offer a proof of ${ }^{64} \mathrm{Cu}^{2+}$ $\mathrm{Fe}^{3+}$-RGD-PEG-MNPs as an impressive simple and natural strategy for tumor imaging and guided resection (Fig. 5A) [56].

A hybrid technique based on the generation of acoustic signals by the detection of optical energy absorption, photoacoustic imaging (PAI), has emerged to overcome the limitations of optical imaging, such as light diffusion. This process consists in the absorption of energy by endogenous chromophores, like $\mathrm{Hb}$ and melanin, that thermoelastically convert the energy into acoustic waves, able to be transduced into a signal [72]. Therefore, specific contrast agents capable of targeting non-absorbing molecules and with strong optical properties are needed. This technique outdoes the previous optical imaging ones in account of the upgraded sensitivity and resolution and the superior achieved depth in the absence of ionizing radiation [58].

For example, $\mathrm{Xu}$ et al. made use of the biocompatibility of MNPs and conjugated them with manganese $\left(\mathrm{Mn}^{2+}\right)$-contrast agents, being $\mathrm{Mn}^{2+}$ also an endogenous constituent of human cells (Fig. 5B). Ultrasmall MNPs were PEGylated to improve their aqueous dispersibility and then chelated with $\mathrm{Mn}^{2+}\left(\mathrm{Mn}^{2+}\right.$-PEG-MNPs), which proved to be strongly bonded, since only $1 \%$ of the $\mathrm{Mn}^{2+}$ was released from the $\mathrm{Mn}^{2+}$-PEG-MNPs within $2 \mathrm{~h}$. Regarding cytotoxicity, the PEG-MNPs both before and after chelating with $\mathrm{Mn}^{2+}$, proved to be safe and biocompatible with Hep-2 or NIH-3T3 cells, which incorporated the $\mathrm{Mn}^{2+}$-PEG-MNPs within their endosomes or lysosomes. Compared to other contrast agents, such as gadolinium, the magnetic gradient was 3.4-fold increased using the $\mathrm{Mn}^{2+}$ PEG-MNPs. After confirming their cellular viability, the $\mathrm{Mn}^{2+}$-PEGMNPs were intravenously injected into tumor-bearing mice. After 30 min of injection, a prominent contrast signal was observed, specifically at the tumor site, in comparison with the surrounding muscle, strongly supporting the tumor targeting effect. After $24 \mathrm{~h}$, the signal started to decrease, probably due to the metabolism and clearance of the $\mathrm{Mn}^{2+}$-PEG-MNPs. $\mathrm{Mn}^{2+}$-PEG-MNPs showed no noticeable toxic effects, being eliminated from the organism after $48 \mathrm{~h}$, thereby avoiding the typical accumulation of these systems [13].

Melanin is an endogenous chromophore that is known to hold photoacoustic (PA) features in vivo, although its sensitivity is limited by its concentration in situ. Hence, an approach to intensify the PA signal was performed by Ju et al. [73], based on the aggregation between MLNPs under specific $\mathrm{pH}$ (pH-MLNPs). MLNPs were produced by oxidation of Da hydrochloride and those were further modified on the surface with the addition of amine groups, specifically mPEG-SH and ethylenediamine (Fig. 5C). After that, citraconic anhydride reacted with the previous amines, forming amide bonds. Under acidotic environment, typical of tumor tissue, the citraconic amide suffered hydrolysis and the prior positive surface charge of the MLNPs changed, enabling the aggregation of the MLNPs by electrostatic interactions, giving rise to pH-MLNPs. This aggregation resulted in an overlapping of the thermal fields, consequentially increasing the PA signal, which may be due to an increase in the rate of the thermal flux. The PA signal was enhanced 8-fold in pH-MLNPs compared to neutral conditions, after $90 \mathrm{~min}$. Those results were comparable with methylene blue and gold nanorods at the same concentrations. After internalization in SKBR3 cells, $\mathrm{pH}-$ MLNPs showed an increase of the PA signal of $84 \%$, compared with dispersed MLNPs (control), phenomenon that can be attributed to the accumulation of pH-MLNPs inside the cells. To assess the be- 


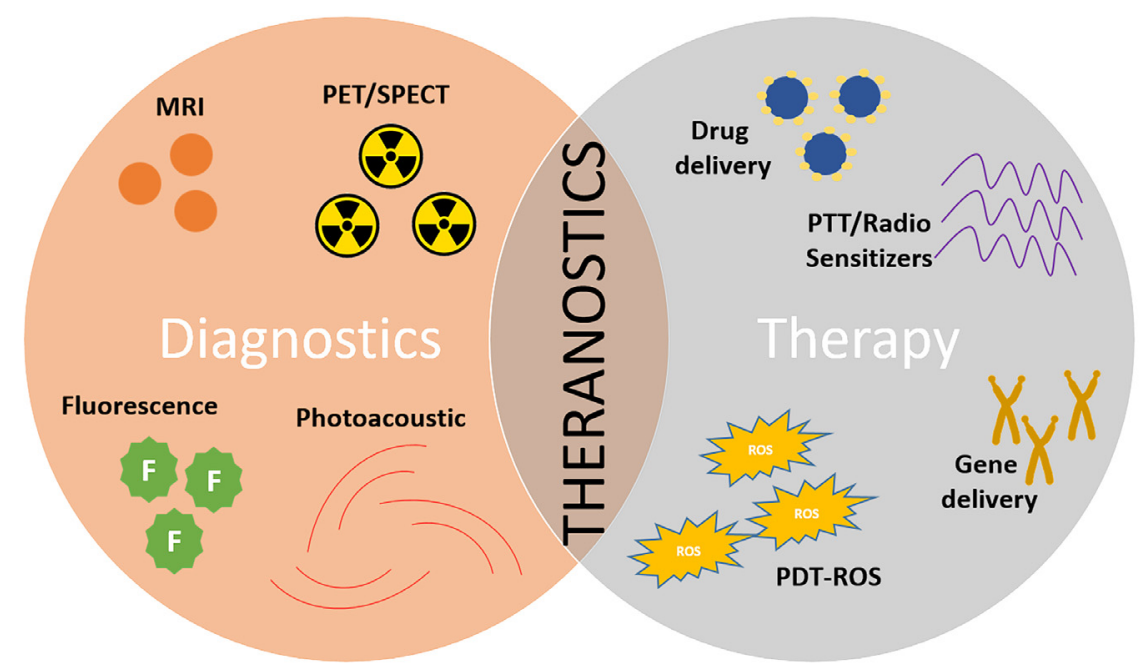

Gadolinium Radiotracer F Fluorophore

Drug delivery molecule

Yomencentem

Fig. 6. Schematic representation of the theranostics approach that gathers in a single system the diagnostic and therapy tools.

havior of pH-MLNPs in vivo, B16 tumor-bearing mice were intravenously injected with $\mathrm{pH}-\mathrm{MLNPs}$. In the mice injected with the control, the PA signal decreased after 30 min post-injection. On the contrary, the PA signal of the mice injected with pH-MLNPs was found to increase up to $2 \mathrm{~h}$ and with an intensity of the signal 2.4-fold higher. Thereby, those pH-MLNPs were presented as an interesting imaging methodology, with superior specificity than the current used methods, without resort to dangerous radiation or cytotoxic contrast agents.

In human body, melanin is distributed among the cells and, although it cannot be used for protein distribution studies, it can be used, as already described, as a contrast agent, due to its strong optical and stability properties. This way, Liopo et al. developed synthetic melanin as a nano contrast agent. It was accounted important factors for in vivo applications of MLNPs regarding two important aspects: concentration, which should be high enough to compete with the RBCs; and the high dispersibility in biological medium. After obtaining the MLNPs, the surface was modified with PEG, to avoid opsonization, granting, simultaneously, stability in sodium chloride ( $\mathrm{NaCl})$, PBS and FBS solutions, with absence of toxicity. The authors also compared their previous work with gold nanorods and observed that the toxicity of MNPs was 2-fold lower than the gold nanorods. In vivo toxicity studies were carried out by intravenous injection of MLNPs in mice and no toxicity or accumulation effects were observed (Fig. 5D). Therefore, melanin proved to be a biodegradable and sustainable optical contrast agent to be used instead of the common metals [12].

\subsection{Theranostics}

Theranostics is the leading approach in recent medicine that combines both specific targeted therapy to cells, or ligands based on specific targeted diagnostic tests, in order to eradicate the pathological condition. It comes from the need to overcome the conventional medicine to a contemporary personalized and precise treatment. Nanotechnology is the basis of this approach that gathers in a single agent the tools for diagnosis, drug delivery and controlled response treatment. Fig. 6 exemplifies the main approaches in which theranostics relies having as basis the diagnostic and therapy approaches currently used.

As previously described, PTT has gained special interest in cancer treatments for being non-invasive, contrary to other therapies currently used. Synthetic materials, like gold nanoparticles, already hold these multifunctional features, however there's a need to use bio materials towards this aim $[74,75]$. Hence, natural melanin extracted from sesame seed was used for sentinel lymph node (SLN) mapping and cancer PTT, assembling the multifunctional features of theranostics. The SLN mapping can inform if the primary tumor is spread or not, being an important tool to acquire better and defined treatment strategies [76].

Thus, MNPs extracted from the seeds were incorporated into liposomes, to improve the dispersion properties (Fig. 7A). The photothermal conversion of the MNPs and liposomes coated-MNPs (LMNPs) was investigated upon exposition to $808 \mathrm{~nm}$ laser irradiation, concluding that both formulations suffered an increase in temperature, with LMNPs reaching a higher temperature but at a lower rate (Fig. 7B i) and ii)). These results were confirmed by in vivo intratumorally injection of LMNPs on tumor-bearing mice (Fig. 7B iii), in which the temperature was raised to ca. $43^{\circ} \mathrm{C}$, within 3 min after irradiation, in the tumors' location. The LMNPs did not exhibit any signs of toxicity upon daylight exposition or in its absence in normal and esophagus carcinoma cells (Eca), contrary to the most used blue dye for SLN mapping, methylene blue (MB), confirming the exceptional biocompatibility of LMNPs. Even though the presence of the LMNPs activated the immune system, leading to an increase of the production of WBCs, it did not change the liver and kidney functions. Experiments performed in normal and tumor-bearing mice showed that after LMNPs injection, these migrated into the SLN in the side where they were injected, attending to its gray color, contrary to the normal color of the lymph node (LN) of the other side, proposing the ability to target the SLN. The safety of the LMNPs was further confirmed by the absence of toxic effects on the major organs, except on the SLN, the LMNPs target, where an inflammatory response was induced on the injected side. To assess the cancer therapy effect of the LMNPs, cancer cells were treated with LMNPs both in the presence and in the absence of laser irradiation, and it was possible to verify that the cell membrane was destroyed only in the presence of irradiation. The results showed that, in absence of radiation, the viability of the cells was close to $100 \%$, while when those were submitted to irradiation, their viability decreased to ca. $27 \%$. Finally, the LMNPs were intratumorally injected into the Eca-109 tumor bearing mice followed by laser irradiation, decreasing the tumor size from 4$5 \mathrm{~mm}$ to almost zero, after 18 days-time period (Fig. 7B iii)). Re- 
A)
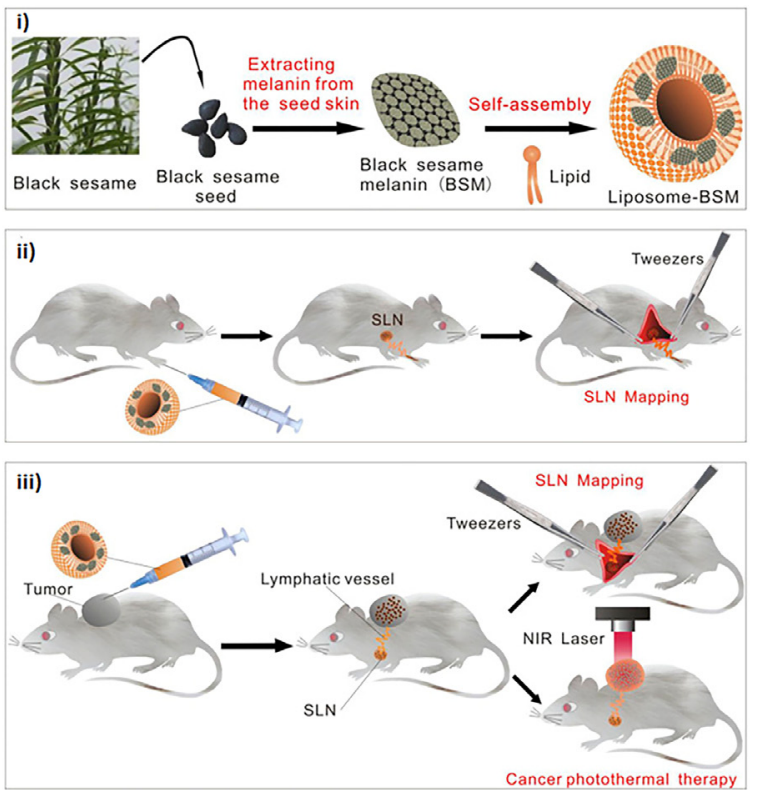
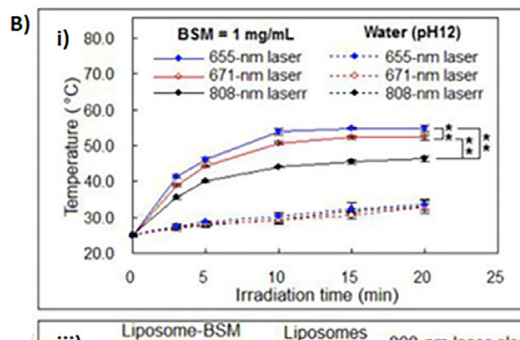

iii) $\begin{gathered}\text { Liposome-BSM Liposomes } \\ \text { 808-nm laser }\end{gathered} \quad 808$-nm laser 808 -nm laser alone

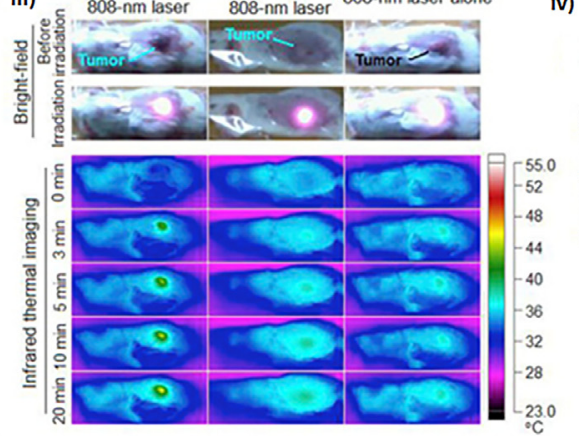

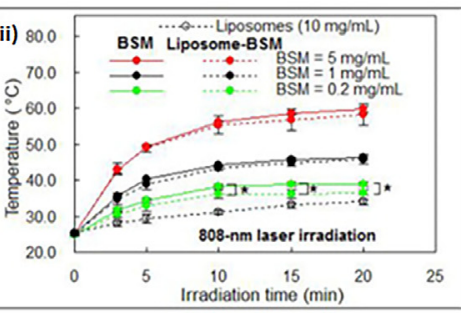

iv)

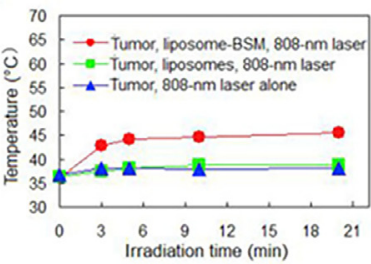

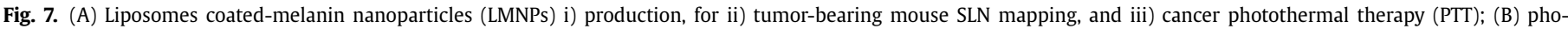

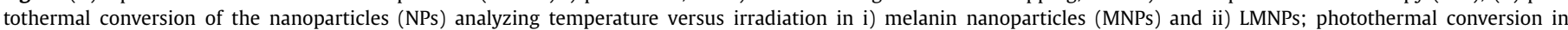

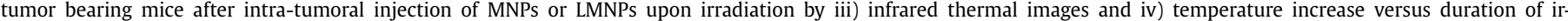

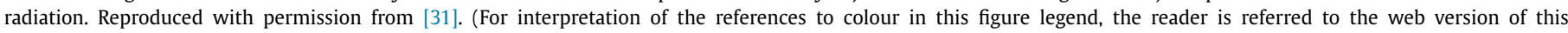
article.)

sults depicted that $5 \mathrm{mg} \cdot \mathrm{mL}^{-1}$ of black sesame melanin was a sufficient concentration for inhibiting tumor growth, due to the raised temperature up to $45{ }^{\circ} \mathrm{C}$ after irradiation, that was capable of destroying the tumor tissue by apoptosis and necrosis (Fig. 7B iv)). Therefore, this kind of melanin is considered safer than the frequently applied $\mathrm{MB}$, presenting no signs of radioactivity, proving its potential use for cancer mapping and treatment [31].

One of the problems observed with nanocarriers arising from the use of these approaches is that they are recognized and excluded by the reticuloendothelial system for being considered as exogenous. In order to tackle this challenge, a solution was found using bio-membranes for the construction of a biocarrier capable of being recognized as endogenous, such as RBC membranes, which surface contains biomarkers such as CD47 proteins, peptides and glycans, that helps escaping from the immune system recognition. Such strategy increased the biocompability and the prolonged retention effect of the nanocarriers in the targeted cells [77].

Jiang et al. [32], used MNPs extracted from cuttlefish and incorporated them into RBC membrane (RBC-MNPs) to use them both for imaging and PTT purposes (Fig. 8A i)). To confirm the photothermal effect, the extracted melanin, was exposed to a NIR laser and the temperature was successfully elevated as the melanin concentration increased. Simultaneously, MLNPs were also synthetically produced and it was verified that natural melanin evidenced a better photothermal activity, compared to the synthetic variant, with conversion efficiencies of $40 \%$ and $29 \%$, respectively. The substantial biodegrability of melanin was studied in the presence of $\mathrm{H}_{2} \mathrm{O}_{2}$, molecules presented in the reticuloendothelial system, specifically in macrophages, presenting a half maximum degradation concentration $\left(\mathrm{DC}_{50}\right)$ much lower for natural in relation to synthetic melanin (0.86 mM versus $4.55 \mathrm{mM}$, respectively). MNPs showed very low toxicity values when incubated in healthy HEK293T and A549 cancerous cells. However, after 5 min irradiation, the viability of A549 cancer cells decreased over 65\%, for a $50 \mathrm{mg} \cdot \mathrm{mL}^{-1}$ concentration of MNPs. In fact, the apoptosis of these cells reached $94 \%$, suggesting a high photothermal effect by the MNPs. Afterwards, RBC-MNPs were prepared by extruding the membrane and melanin through a porous membrane, which was successfully correlated with a $16 \mathrm{~nm}$ increase of NPs size. The resulting RBC-MNPs were capable to retain the same photothermal properties as MNPs. Regarding pharmacokinetics, after 24 h postintravenous injection of RBC-MNPs or MNPs, a concentration of $11.16 \%$ contrasting with ca. $1 \%$ was detected in blood circulation, respectively. These results suggest that the RBC membrane was incorporated into the surface of MNPs allowing their escape towards the immune system. The biodistribution studies revealed a 1.44fold increase tumor accumulation of RBC-MNPs following $4 \mathrm{~h}$ injection, compared to MNPs, confirming the camouflage capabilities of RBC coating. The tumor accumulation efficiency of the RBCMNPs was assessed by photoacoustic imaging, depicting a 1.35 -fold accumulation enhancement of RBC-MNPs compared with MNPs at $4 \mathrm{~h}$ post- intravenous injection, which was probably due to the escape capabilities from the immune system (Fig. 8A ii and iii)). After $24 \mathrm{~h}$, the PA effect dropped, owing to the degradation of melanin by tumor cells. Finally, A549 tumor-bearing mice were subjected to PTT and RBC-MNPs were able to destroy the tumor within 13 days, leading to an antitumor rate of almost $100 \%$. On the other hand, whilst tumors in RBC-uncoated MNPs plus radiation group allowed the tumor re-grow after treatment, probably due to the lower tumor accumulation, despite the antitumor rate of ca. $78 \%$, leading to an antitumor rate of almost $100 \%$ for RBC-MNPs, compared to MNPs. Also, the renal and hepatic functions as well as blood parameters were not affected, confirming the safety of the RBC-MNPs. In this study is important to highlight the differences observed with natural MNPs and MLNPs, specifically in terms of superior photothermal effect against cancer cells and the lower $\mathrm{DC}_{50}$ observed for MNPs that translates in superior biodegradability. These results indicate that natural melanin could be a better option to be used in PTT approaches.

In another approach, PDa was used as MLNPs for multimodal imaging and cancer therapy, by using PTT. These MLNPs were further chelated with $\mathrm{Fe}^{3+}$, and indocyanine green (ICG), a NIR dye, to enhance the low absorption coefficient of PDa in NIR $\left(\mathrm{Fe}^{3+}\right.$ ICG-MLNPs). Further, mPEG-SH was used to cover the previous MLNPs, in order to enhance their stability. The incorporation of 
A) i)

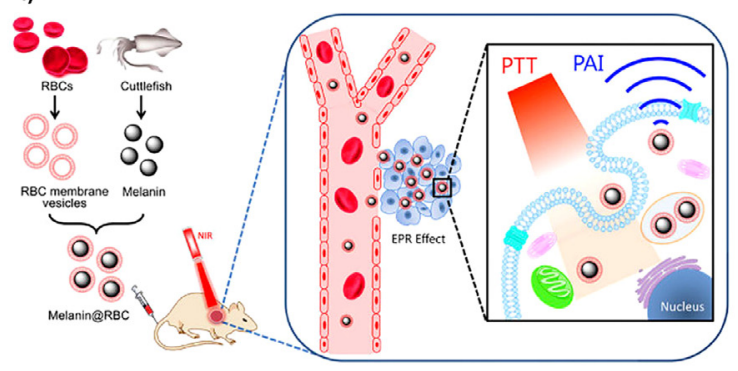

B) i)

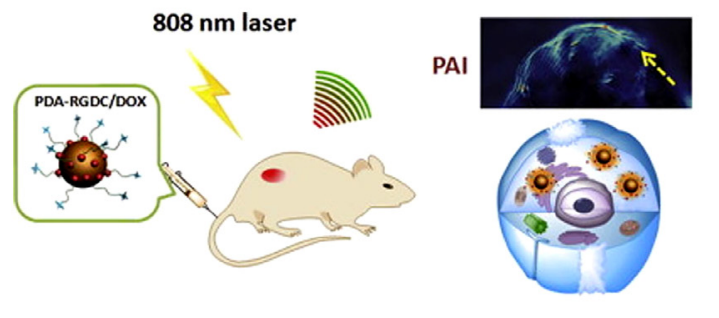

Chemo-PTT synergistic therapy ii)

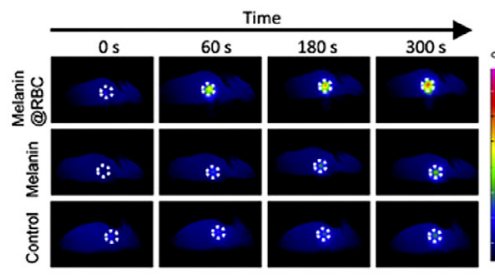

iii)

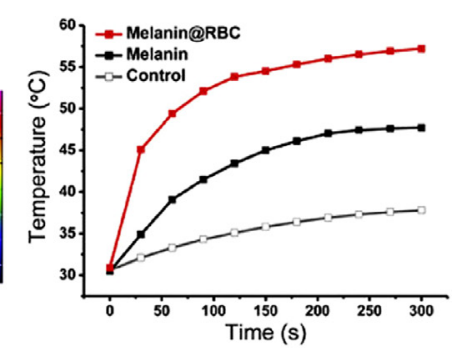

ii)

Bs

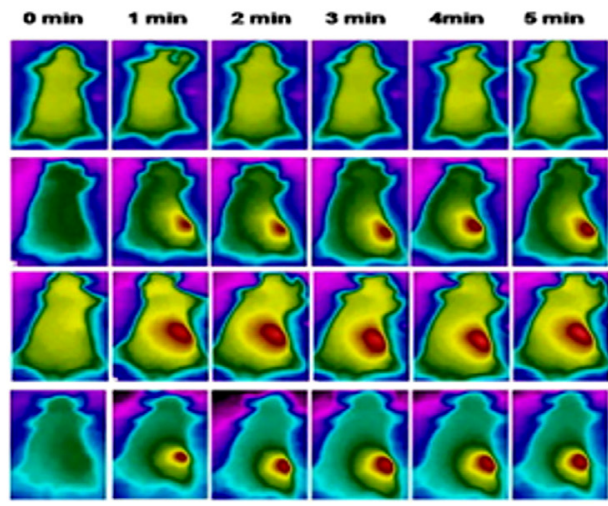

+ Laser

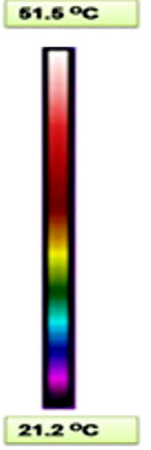

PDA-RGDCRDOX

Dox

$\operatorname{logx}$

POA POA Rooc

POA-RoOC
hoOx LLaser

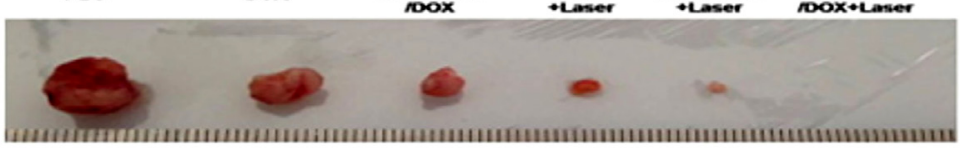

ii)
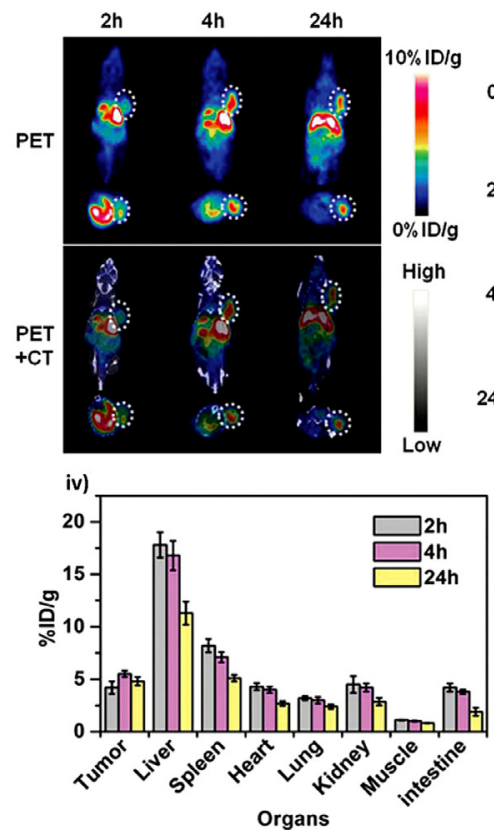
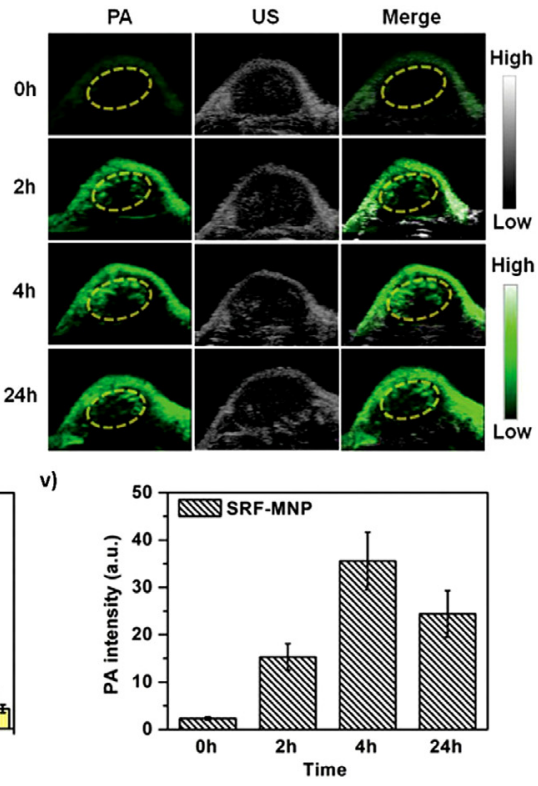

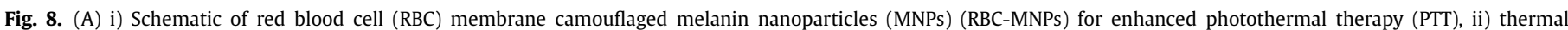

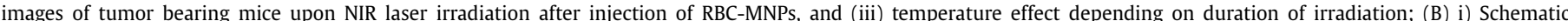

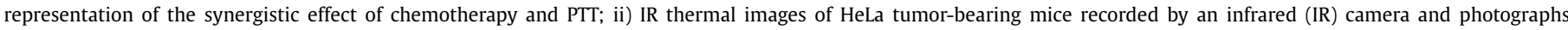

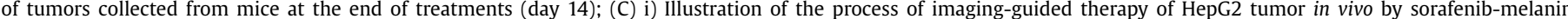

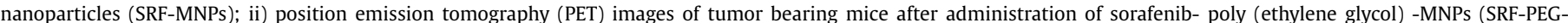

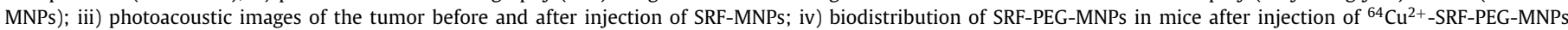

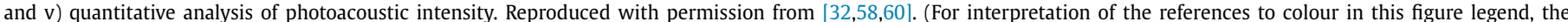
reader is referred to the web version of this article.) 
ICG increased by ca. 6-times the optical absorption of $\mathrm{Fe}^{3+}$-ICGMLNPs compared to $\mathrm{Fe}^{3+}$-MLNPs. Nevertheless, it decreased the fluorescence intensity of $\mathrm{Fe}^{3+}$-MLNPs, which turns to be an advantage, since the photothermal conversion by nonradioactive decay would increase the PTT efficiency. The photothermal effect was much higher in the $\mathrm{Fe}^{3+}$-ICG-MLNPs, as it raised their temperature to $55.4{ }^{\circ} \mathrm{C}$ upon irradiation. Photoacoustic and MRI signals were also enhanced by the presence of ICG and $\mathrm{Fe}^{3+}$, respectively, in the MLNPs surface. The biocompatibility of the $\mathrm{Fe}^{3+}$-ICG-MLNPs was successfully confirmed by incubation with three different cell lines (4T1, 293T and H460), with no signs of cytotoxicity after $24 \mathrm{~h}$. When 4T1 cells were incubated with MLNPs and $\mathrm{Fe}^{3+}$-ICG-MLNPs after irradiation, a significant difference was observed, namely only $35 \%$ cells were killed compared to $96 \%$, respectively. No noteworthy cell death was observed with MLNPs or only laser irradiation (controls), which once again sustains their safety and stability. In vivo experiments were conducted in 4T1 tumor-bearing mice, which were intravenously injected with the $\mathrm{Fe}^{3+}$-ICG-MLNPs and submitted to PA and MRI imaging. After $24 \mathrm{~h}$, a substantial enhancement was detected in tumor region, which might be due to the EPR effect. At the same time, MRI results showed that the signal was enhanced by 25 -fold after $24 \mathrm{~h}$, compared to results before intravenous injection. This dual mode imaging is of great promise and makes cancer results much more accurate and specific than the currently used. Histology and hematology analysis of collected tumor tissue showed that only the laser irradiation treated groups presented tumor necrosis. MLNPs with irradiation group triggered karyolysis (dissolution of the cell nucleus), however an extensive cancer necrosis was achieved using $\mathrm{Fe}^{3+}$-ICGMLNPs with laser irradiation, emphasizing the lack of efficiency of the remaining tested formulations. Finally, the tumor inhibition was assessed through the growth rate of tumors, where a regrow of the cancer cells after 3 days, with consequential animal death, was observed in the MLNPs treated with laser irradiation. However, the growth of the tumor was completely inhibited with the $\mathrm{Fe}^{3+}$-ICG-MLNPs, which was consistent with the $100 \%$ animal survival rate using these NPs, denoting remarkable promises for clinical application [57].

Following the same strategy, MLNPs using Da hydrochloride were synthetized, but in this work using an arginine - glycine - aspartic - cysteine acid (RGDC) peptide to target cancer cells, facing its recognized affinity to the $\alpha \mathrm{v} \beta 3$ integrins located in cancer cells (RGDC-MLNPs). Simultaneously, DOX was attached into the MLNPs surface in order to be stimuli-responsive released, specifically upon NIR irradiation (DOX-RGDC-MLNPs). To upgrade the therapeutics, PTT was used to additionally improve the efficacy of the treatment. Fig. 8B i) schematically represents the approach that researchers followed. The release profile of DOX from DOX-RGDC-MLNPs evidenced a noticeable burst stage related to the NIR irradiation. This behavior was attributed to the destructed intermolecular forces formed between Da and DOX. This proves to be a relevant advantage, since only in the presence of cells lysosome and irradiation occurs the drug release, granting the safety of the treatment. In vitro tests showed that DOX-RGDC-MLNPs were more able to be internalized by HeLa cells, specifically in the nucleus, than DOXMLNPs, confirming the capability of the RGDC to target tumor integrins. After laser irradiation, DOX was greatly released from the DOX-RGDC-MLNPs, relatively to control experiments, accompanied by extensive cell death. Noteworthy, DOX-RGDC-MLNPs were able to improve the treatment efficacy compared to PTT or chemotherapy used as individual treatments in such a way that the cell viability was $84 \%$ and $72 \%$ for MLNPs and RGDC-MLNPs under photothermal treatment, respectively, and $55 \%$ and $48 \%$ for DOX and DOX-RGDC-MLNPs without irradiation. Differently, under NIR irradiation, DOX-RGDC-MLNPs were capable to diminish the cells viability to $22 \%$ [58].
The PAI ability of the produced DOX-RGDC-MLNPs was assessed in $\alpha \mathrm{v} \beta 3$ integrin HeLa tumor-bearing mice, and those structures proved to be strong imaging agent as it delineated the margins of the tumor with optimum signal at $8 \mathrm{~h}$ post-intravenous injection. Moreover, this ability was studied in additional tumor types, as $4 \mathrm{~T} 1$ bearing mice, which considerably increased 3-fold the PA signal contrary to the use of single MLNPs. These findings suggest the potential use of DOX-RGDC-MLNPs as a nanoimaging agent for tumor theranostics. Finally, the multi-modal therapeutics was tested in HeLa nude tumor-bearing mice. Mice treated with DOXRGDC-MLNPs without radiation and RGDC-MLNPs under irradiation showed that the inhibition of tumor growth was only delayed, as it started regrowing after two days. Contrary, DOX-RGDC-MLNPs under irradiation inhibited the tumor growth (Fig. 8B ii)), completely eradicating the tumor within 50 days. This study clearly evidences that the combined use of two referred therapies are able of eradicating the tumor by PTT, using heat to directly destroy them, and chemotherapy, which drug release is also stimulated by heat. Thus, it is possible to prevent the regrowth of the tumor, which has been one of the major drawbacks of current chemotherapeutics [58].

Zhang et al. continued their previous work [56], which consisted in the use of MNPs as a biomarker for tumor imaging, but in this case as using the same MNPs as a drug-delivery system. Therefore, sorafenib (SRF), a multikinase inhibitor drug used for unresectable hepatocellular carcinoma treatment, was incorporated into sorafenib-poly (ethylene glycol)-MNPs (SRF-PEG-MNPs) (Fig. 8C i)). Drug release studies were performed using a dialysis bag, and SRFPEG-MNPs showed an initial burst release of $18 \%$, followed by a gradual release of SRF during $24 \mathrm{~h}$ up to $80 \%$ in both $\mathrm{pH}=7.2$ and 5.0. This profile demonstrates the ability of controlled drug release even in a typical acidic tumor environment. In vitro cytotoxicity assays were carried out in NIH-3T3 and HepG2 cells and the cell viability was found to be maintained in the presence of PEG-MNPs. Contrarily, SRF-PEG-MNPs were cytotoxic against HepG2 cells, confirming the nanocarrier capability of the SRF-PEG-MNPs. As previously described, MNPs can be used as a multimodal imaging agent, not only for acquire information about the biodistribution of the drug delivery system using PET, but also for information about the biodistribution in the tumor using PAI [56]. Hence, to monitor the SRF delivery using PET, ${ }^{64} \mathrm{Cu}^{2+}$ was incorporated into SRF-PEGMNPs $\left({ }^{64} \mathrm{Cu}^{2+}\right.$-SRF-PEG-MNPs). After tail-vein injection of ${ }^{64} \mathrm{Cu}^{2+}$ SRF-PEG-MNPs on HepG2-tumor-bearing mice the highest tumor uptake was achieved after $4 \mathrm{~h}$, corresponding to $20 \%$ of SRF released, followed by a continuous accumulation of the NPs and a consequent steady release of SFR, where only ca. $13 \%$ of ${ }^{64} \mathrm{Cu}^{2+}$ SRF-PEG-MNPs were lost after 24 h post-injection Fig. $8 \mathrm{C}$ ii)). The radioactivity of ${ }^{64} \mathrm{Cu}^{2+}$ was detected only in the liver and spleen, which shows that the excretion occurred through the hepatobiliary system Fig. 8C iv)). In solution, PA signal was almost the same for PEG-MNPs and SRF-PEG-MNPs, confirming that SRF doesn't affect the PAI properties of MNPs. PAI was used to study the behavior of the SRF-PEG-MNPs in the tumor, so PA signals were detected $2 \mathrm{~h}$ after tail-vein injection in the superficial area of the tumor, reaching its maximum at $4 \mathrm{~h}$. However, at $24 \mathrm{~h}$ the surface signal was decreased, while the whole tumor appeared to have a signal, showing that the NPs were internalized by the tumor Fig. 8C iii) and v)). After 20 days post-treatment, in control groups (PBS and PEG-MNPs treated group), the tumor continued to grow, contrary to SRF-PEG-MNPs group, which were able to inhibit the tumor growth with no loss of body weight, compared with the oral free SRF administrated group. These results make proof that MNPs may be a safer system for SRF tumor delivery with higher efficacy, as the necrosis of tumor cells was superior in SRF-PEG-MNPs administrated group than in oral free SRF administrated group. Additionally, these SRF-PEG-MNPs possibly the diminishment of the SRF dosage necessary to inhibit the tumor growth to $4 \mathrm{mg} \cdot \mathrm{kg}^{-1}$, 
compared to the higher dosage needed using oral free SRF, specifically $20 \mathrm{mg} \cdot \mathrm{kg}^{-1}$ [60].

Iron oxide nanoparticles (IONPs) have been used for imaging diagnostics and therapeutics due to their magnetic properties and biodegradation, through endosomes, after cell internalization [78]. Wang et al. prepared MNPs with $\mathrm{Fe}_{3} \mathrm{O}_{4}\left(\mathrm{Fe}_{3} \mathrm{O}_{4}-\mathrm{MNPs}\right)$ by a co-precipitation method in order to use them for MRI, PAI and PPT. The photothermal effect was higher when a concentration of $10 \mathrm{mM}$ of $\mathrm{Fe}_{3} \mathrm{O}_{4}$-MNPs was used, allowing for a temperature increase of approximately $40{ }^{\circ} \mathrm{C} . \mathrm{Fe}_{3} \mathrm{O}_{4}$-MNPs showed also a high magnetic behavior, which was shown to increase with the concentration of $\mathrm{Fe}_{3} \mathrm{O}_{4}$-MNPs, just like the PA signal behavior. Comparatively to IONPs available in the market, $\mathrm{Fe}_{3} \mathrm{O}_{4}-\mathrm{MNPs}$ surpass not only the absorption in the NIR region by the photothermal effect, which can be attributed to the presence of MNPs. In vitro studies were conducted in human U-87 MG cells to evaluate the MRI and PA signals of $\mathrm{Fe}_{3} \mathrm{O}_{4}$-MNPs. These NPs showed a superior contrast for MRI as the concentration of $\mathrm{Fe}_{3} \mathrm{O}_{4}$-MNPs increased, such as PAI. No cytotoxicity effects were visible, even using high concentrations: $100 \mu \mathrm{g}$ Fe.mL $\mathrm{mL}^{-1}$, confirming the safety and stability of the produced $\mathrm{Fe}_{3} \mathrm{O}_{4}-\mathrm{MNPs}$. Nevertheless, when cells were irradiated with NIR, along with the presence of $\mathrm{Fe}_{3} \mathrm{O}_{4}-\mathrm{MNPs}$, the cellular viability was decreased to $43 \%$. U-87 MG tumor-bearing mice were interstitially injected with $\mathrm{Fe}_{3} \mathrm{O}_{4}$-MNPs and subjected to PAI and the results exhibited a high signal after 5 min post-injection, which was maintained to $1 \mathrm{~h}$ post-injection. Regarding MRI, the signal intensity increased over time, similar to PAI, which depicted a regular distribution of the $\mathrm{Fe}_{3} \mathrm{O}_{4}-\mathrm{MNPs}$ through the tumor tissue with time. These imaging techniques are crucial to determine the exact location of the tumor and to proceed with the subsequent treatment. Finally, PTT was conducted into the U-87 MG tumor-bearing mice divided in four groups: no treatment control group; laser control group (only subjected to $808 \mathrm{~nm}$ of laser irradiation); $\mathrm{Fe}_{3} \mathrm{O}_{4}-\mathrm{MNPs}$ group (tumor injection with $\mathrm{Fe}_{3} \mathrm{O}_{4}-\mathrm{MNPs}$ without laser irradiation); $\mathrm{Fe}_{3} \mathrm{O}_{4}-\mathrm{MNPs}$ and irradiation group (tumor injection with $\mathrm{Fe}_{3} \mathrm{O}_{4}-\mathrm{MNPs}$ and $808 \mathrm{~nm}$ of laser irradiation). The temperature of the tumor was elevated up to $20^{\circ} \mathrm{C}$, contrary to no treatment and laser control groups. The tumor volume was measured and, after 15 days post-treatment, there were no signs of tumor regrowth in the $\mathrm{Fe}_{3} \mathrm{O}_{4}$-MNPs and irradiation treated group which, was found to be eliminated, contrasting with no tumor growth inhibition on the remaining groups. Nonetheless, no changes were observed in the major organs, neither adverse effects. Gathering these results, $\mathrm{Fe}_{3} \mathrm{O}_{4}$-MNPs have shown to be an interesting and safe agent to use in imaging and treatment of glioblastoma [33].

Similar to the previous study, Cho et al. coated MLNPs with silica $\left(\mathrm{SiO}_{2}\right)$, to improve the biocompatibility, aqueous dispersibility and made a surface functionalization by using additional ligands. As a metal ion, the authors applied gadolinium $\left(\mathrm{Gd}^{3+}\right)$ to use as a dual modal contrast agent in MRI and fluorescent imaging. MLNPs were immersed in a $\mathrm{Gd}^{3+}$ solution to obtain $\mathrm{Gd}^{3+}$-MLNPs. Then, $\mathrm{SiO}_{2}$ coating was added to the previously referred MLNPs $\left(\mathrm{SiO}_{2}-\right.$ $\mathrm{Gd}^{3+}$-MLNPs), whose process changed their surface charge from positive to negative, providing a higher dispersibility of these NPs. The magnetic properties of $\mathrm{SiO}_{2}-\mathrm{Gd}^{3+}$-MLNPs were confirmed by an increase in the magnetization upon application of a magnetic field. For fluorescent imaging application, $\mathrm{SiO}_{2}-\mathrm{Gd}^{3+}-\mathrm{MLNPs}$ were labeled with tetramethylrhodamine (TRITC) (TRITC-SiO ${ }_{2}-\mathrm{Gd}^{3+}$ MLNPs), which fluorescent properties of TRITC were enhanced by the increase in $\mathrm{SiO}_{2}$ coating. The presence of $\mathrm{SiO}_{2}$ avoids the quenching effects of quinone residues in MLNPs that capture the excited electrons of fluorescent TRITC molecules. The photothermal properties of the $\mathrm{SiO}_{2}-\mathrm{Gd}^{3+}$-MLNPs were very positive, as the temperature was enhanced up to $57{ }^{\circ} \mathrm{C}$, using a concentration of $1.2 \mathrm{mg} \cdot \mathrm{mL}^{-1} \mathrm{SiO}_{2}-\mathrm{Gd}^{3+}$-MLNPs. The biocompability of $\mathrm{SiO}_{2}-\mathrm{Gd}^{3+}$
MLNPs was studied in human prostate cancer cell line (PC-3) where the coating of $\mathrm{SiO}_{2}$ was able to improve the cellular viability, compared to $\mathrm{Gd}^{3+}$-MNPs, specifically $70 \%$ and $51 \%$, respectively. In vitro photothermal studies were also conducted in PC-3 cell lines treated with $\mathrm{SiO}_{2}-\mathrm{Gd}^{3+}$-MLNPs. When NIR irradiation was applied, significant cell death was observed. In vivo tests were conducted in PC-3 tumor bearing-mice and, after confirming the tumor location by MRI, TRITC-SiO $2-\mathrm{Gd}^{3+}$-MLNPs were intratumorally injected. MRI showed the deposition of the TRITC-SiO $2-\mathrm{Gd}^{3+}$-MLNPs in the tumor, with an intensification of the signal by 9-fold and a strong fluorescence where the NPs were located, evidencing an increase of 14-fold, compared to normal tissue. After the multimodal imaging, the tumor was irradiated with NIR, and the temperature raised up to $60{ }^{\circ} \mathrm{C}$ after $2 \mathrm{~min}$. It was noticeable that there was necrosis of tumor tissue without damaging viable cells, confirmed by tissue staining. Furthermore, apoptosis of tumor cells was confirmed by the significant expression of a biomarker responsible by stress induced by heat, the heat-shock protein 70 . By the aforementioned premises, TRITC- $\mathrm{SiO}_{2}-\mathrm{Gd}^{3+}$-MLNPs are identified with a great promise to use in theranostics [59].

The newest therapies are becoming more non-invasive, with less dosages to be effective, which makes theranostics an incredible approach for that purpose. Incorporating in a single carrier an all-in-one mechanism, capable of diagnosis and therapy, with guarantee of treatment efficacy and safety constitutes the focus of the future science.

Melanin has inherent features that make it adequate for imaging, such as photoacoustic and by surface functionalization with ligands, permitting its use with common imaging techniques, like MRI and PET. On the other hand, the PTT effect of melanin showed to be very promising, once allied to a drug improves remarkably the efficacy of the treatment. Therefore, putting together imaging techniques, which can determinate the exact location of the nanocarrier and effective targeted treatments, constitutes a successful strategy of growing biomedical interest.

\subsection{Bioengineering and bioelectronics}

Melanin may, not only be used as a nanocarrier for parenteral administration, but also for the emerging bioengineering, for instance for implantable devices and bionic materials, due to its inner conductive and metal chelating and biocompatibility properties [79].

MNPs were assembled with poly (vinyl alcohol) (PVA) to form a nanocomposite film with a thickness of, approximately, $300 \mathrm{~nm}$ (PVA-MNPs). The electric properties of the film were assessed on an indium tin oxide (ITO) electrode, which was capable to increase the charge storage capacity by 1000 cyclic voltammetry of the bare ITO. Furthermore, biocompatibility was evaluated in murine macrophage cell lines and compared to the bare ITO. Results revealed that not only PVA-MNPs films induced no cell damaged, but also were able to reduce the secretion of inflammatory cytokines. When lipopolysaccharide was added to the culture medium, the induced inflammatory responses were reduced by 5-fold, when PVA-MNPs films were incorporated into the ITO. Also, PC 12 cells were cultured in the produced films, with an observable neural cell differentiation, which can be due to the electrical conductivity of the PVA-MNPs films. This constitutes a major step for bioengineering in ways that a biomaterial can be used into implantable devices and electroceuticals, with pronounced improved features, in relation to the common used materials [26].

Regarding wound healing conventional approaches, the majority of the materials used are gauzes, hydrogels and foams that are applied to prevent antimicrobial infections and lead to a clean wound healing process. However, at inner wounds, where the microenvironment is moistier than at the outside, less materials, which 
can be used as bio adhesives, are available. Recently, it was found that catechol groups, found in melanin-like materials, can improve bioadhesion of certain materials composed with amine groups [80]. Therefore, MLNPs were synthetized to obtain surgical membranes with capabilities of enhancing the bioadhesion of materials. Firstly, MLNPs biocompability was assessed by the lactate dehydrogenase (LDH) assay, by using primary dermal fibroblasts, which viability was affected only with high concentrations of MLNPs. Also, the produced MLNPs exhibited antibacterial effects against gram negative and positive bacteria, which could be attributed to the interaction with the bacteria membranes, leading to their death. In addition, MLNPs were incorporated into polysaccharide basedmembranes and their adhesion was studied in the external part of porcine intestine, a very moisty part of the lumen, in a Hank's Balanced Salt Solution (HBSS). The detachment of the membranes was evaluated, being observable that non-functionalized MLNPs membranes detached after $90 \mathrm{~min}$, contrary to the MLNPs-incorporated ones, which were able to stay in place up to $24 \mathrm{~h}$. The production of this kind of biomaterial is very important for the new generation of green nanosystems, with marked improved effects regarding biocompability and biosafety [61].

Concerning bioelectronics, one of its challenges is connect biological signals (e.g. ionic signals) with conventional electronics, allowing the creation of new medical therapies. In order to overcome this drawback Sheliakina et al. [62] developed a transducing interface based on the melanin conductivity and a p-type organic electrochemical transistor. Melanin was synthesized and used for film casting. The developed device presented a channel length of $100 \mathrm{~mm}$, ON/OFF ratios as high as 104 and operates at $01 \mathrm{~V}$. The transducer operated by a direct injection of protons into the semiconductor channel- 'volumetric gating' - which modulates the channel current, a necessary requirement for miniaturization. With this study, a functioning electronic device based on melanin was achieved, with fast response time and lower power operation. Furthermore, a significant advance on the pathway that can compute at the biology-electronic interface was achieved.

\section{Concluding remarks and future perspectives}

Melanin, an L-DOPA-derived biopolymer present in wide variety of organisms, evidences, outstanding physicochemical properties that have revealed to be very useful in the medical nanotechnology field. In this comprehensive review, it was discussed the current research on MNPs and MLNPs that are being used for the different biomedical applications, such as antioxidant applications, drug release, imaging, bioelectronics and theranostics. The last one is the new goal of medicine, which aims to incorporate, all-inone, the diagnostic and the therapy. The results are very promising, leading to a new era of medicine, the personalized medicine where nanotechnology is the main pillar. Along with that, the need to find green materials has been very important for the human health technology, and melanin presents inherent meritorious visages for being sustainable and innovative, specifically if it derives from natural sources.

Not only natural melanin but also PDa have been exploited, since those materials have the same precursors and equivalent biocompatibility. Even though their structural similarity, the importance of having relevant physicochemical properties, such as melting point, freezing point, surface tension, water solubility and synthesis procedures that could compare the analogous materials is a challenge that needs to be achieved among the community of scientists. In order to obtain concise relationships between structures, properties and function, deep studies must be undergone using melanin and PDa, both obtained from diverse sources, and through different synthesis procedures, respectively.
While the research is confirming the potentiality of melanin nanocarriers, procedures for obtaining natural melanin and its purifying processes is still lacking, probably due to the many different sources, imposing the unmet need to standardize these processes.

Depending on the cellular target, melanin nanocarriers can be effectively functionalized with specific ligands, avoiding, this way, the major limitations of the actual used carriers: cytotoxicity, accumulation, biodegrability and response time. It was shown that most of the studies incorporated ligands to the MNPs surface in order to achieve better specificity. The functionalization of the MNPs not only increases specificity but also adds features that those in their natural state do not own, such as magnetic properties or anticancer properties. This possibility can represent a chance to use this material as a preset nanostructure, combining the imaging properties of melanin with the therapy properties of particular agents, such as anticancer drugs. By imaging in situ, it is possible to analyze if the targeting was successful and proceed with the modulated release of an agent at the target (e.g., MNPs functionalized with specific hepatocytes ligands under certain stimulus release an anticancer drug along with PTT to destroy the cancer cells). To build such a preset nanocarrier, a release method has to be selected to fit in the diverse environments. In fact, cancer therapy is one of the most studied areas, with a great potential results, using based-melanin carriers. Herein, using the hybrid ionicelectronic conductance properties of melanin, an electrical stimulation for controlled release can be applied to promote the agent delivery specifically in the desired target. Gathering all the knowledge that was herein discussed, the use of melanin as a nanocarrier seems very promising, as it meets all the features important for targeting specific needs. This green economical material leads biomedicine to a new era of personalized treatment achieving improved results with minimized toxicity and increased biostability, with less disadvantages and side effects of the current ones.

\section{Declaration of Competing Interest}

There are no conflicts of interest to declare.

\section{Acknowledgments}

VM Correlo would like to acknowledge FROnTHERA (NORTE-010145-FEDER-0000232) project. R Rebelo would like to acknowledge BREAST-IT PTDC/BTM-ORG/28168/2017 project.

\section{References}

[1] I.A. Siddiqui, V. Sanna, N. Ahmad, M. Sechi, H. Mukhtar, Resveratrol nanoformulation for cancer prevention and therapy, Ann. N. Y. Acad. Sci. 1348 (1) (2015) 20-31

[2] S. Suri, G. Ruan, J. Winter, C.E Schmidt, Microparticles and nanoparticles, In Biomaterials Science (2013).

[3] Y. Nakamura, A. Mochida, P.L. Choyke, H. Kobayashi, Nanodrug delivery: is the enhanced permeability and retention effect sufficient for curing cancer? Bioconjug. Chem. 27 (10) (2016) 2225-2238.

[4] M.C. Bonferoni, S. Rossi, G. Sandri, F. Ferrari, Nanoparticle formulations to enhance tumor targeting of poorly soluble polyphenols with potential anticancer properties, Semin. Cancer Biol. 46 (2017) 205-214.

[5] S. Mura, P. Couvreur, Nanotheranostics for personalized medicine, Adv. Drug Deliv. Rev. 64 (13) (2012) 1394-1416.

[6] F. Mottaghitalab, M. Farokhi, M.A. Shokrgozar, F. Atyabi, H. Hosseinkhani, Silk fibroin nanoparticle as a novel drug delivery system, J. Control. Release 206 (2015) 161-176.

[7] S. Hossen, M.K. Hossain, M.K. Basher, M.N.H. Mia, M.T. Rahman, M.J. Uddin, Smart nanocarrier-based drug delivery systems for cancer therapy and toxicity studies: a review, J. Adv. Res. (2018).

[8] R. Mo, Z. Gu, Tumor microenvironment and intracellular signal-activated nanomaterials for anticancer drug delivery, Mater. Today 19 (5) (2016) 274-283.

[9] J.F. Coelho, P.C. Ferreira, P. Alves, R. Cordeiro, A.C. Fonseca, J.R. Góis, M.H. Gil, Drug delivery systems: advanced technologies potentially applicable in personalized treatments, EPMA J 1 (1) (2010) 164-209.

[10] S. Bhatia, Natural polymer drug delivery systems: nanoparticles, plants, and algae, in: S. Bhatia (Ed.), Natural Polymer Drug Delivery Systems: Nanopar- 
ticles, Plants, and Algae, Springer International Publishing, Switzerland, 2016, pp. 1-225. 2016.

[11] F. Solano, Melanin and melanin-related polymers as materials with biomedical and biotechnological applications-cuttlefish ink and mussel foot proteins as inspired biomolecules, Int. J. Mol. Sci. 18 (7) (2017).

[12] A. Liopo, R. Su, A.A. Oraevsky, Melanin nanoparticles as a novel contrast agent for optoacoustic tomography, Photoacoustics 3 (1) (2015) 35-43.

[13] W. Xu, J. Sun, L. Li, X. Peng, R. Zhang, B. Wang, Melanin-Manganese nanoparticles with ultrahigh efficient clearance in vivo for tumor-targeting T1 magnetic resonance imaging contrast agent, Biomater. Sci. 6 (1) (2017) 207-215.

[14] Q. Fan, K. Cheng, X. Hu, X. Ma, R. Zhang, M. Yang, X. Lu, L. Xing, W. Huang, S.S. Gambhir, et al., Transferring biomarker into molecular probe: melanin nanoparticle as a naturally active platform for multimodality imaging, J. Am. Chem. Soc. 136 (43) (2014) 15185-15194.

[15] P. Meredith, T. Sarna, The physical and chemical properties of eumelanin, Pigment Cell Res 19 (6) (2006) 572-594.

[16] A.B. Mostert, B.J. Powell, F.L. Pratt, G.R. Hanson, T. Sarna, I.R. Gentle, P. Meredith, B.M. Hoffman, Role of semiconductivity and ion transport in the electrical conduction of melanin, Proc. Natl. Acad. Sci. 109 (23) (2012) 8943-8947.

[17] L.F. Wang, J.W. Rhim, Isolation and characterization of melanin from black garlic and sepia ink, LWT 99 (2019) 17-23.

[18] R. Hou, X. Liu, K. Xiang, L. Chen, X. Wu, W. Lin, M. Zheng, J. Fu, Characterization of the physicochemical properties and extraction optimization of natural melanin from inonotus hispidus mushroom, Food Chem 277 (2019) 533542.

[19] T.H. Nasti, L.M.C. Timares, Eumelanin and pheomelanin: their role in determining the susceptibility to skin cancer, Photochem. Photobiol. 91 (1) (2015) $188-200$.

[20] A. Büngeler, B. Hämisch, O.I. Strube, The supramolecular buildup of eumelanin: structures, mechanisms, controllability, Int. J. Mol. Sci. 18 (9) (2017).

[21] M. d'Ischia, K. Wakamatsu, F. Cicoira, E. Di Mauro, J.C. Garcia-Borron, S. Commo, I. Galván, G. Ghanem, K. Kenzo, P. Meredith, et al., Melanins and melanogenesis: from pigment cells to human health and technological applications, Pigment Cell Melanoma Res 28 (5) (2015) 520-544.

[22] L. Huang, M. Liu, H. Huang, Y. Wen, X. Zhang, Y. Wei, Recent advances and progress on melanin-like materials and their biomedical applications, Biomacromolecules 19 (6) (2018) 1858-1868.

[23] National Center for Biotechnology Information. 2018. PubChem compound database https://pubchem.ncbi.nlm.nih.gov/compound/6325610 (Accessed 19 November 2018)

[24] G. Marucci, A. Beeby, Parker, A.W. Nicholson, Raman spectroscopic library of medieval pigments collected with five different wavelengths for investigation of illuminated manuscripts, Anal. Methods 10 (2018) 1219-1236.

[25] A. De Trizio, P. Srisuk, R.R. Costa, A.G. Fraga, T. Modena, I. Genta, R. Dorati, J. Pedrosa, B. Conti, V.M.; Correlo, et al., Natural based eumelanin nanoparticles functionalization and preliminary evaluation as carrier for gentamicin, React. Funct. Polym. 114 (2017) 38-48.

[26] T. Eom, K. Woo, W. Cho, J.E. Heo, D. Jang, J.I. Shin, D.C. Martin, J.J. Wie, B.S. Shim, Nanoarchitecturing of natural melanin nanospheres by layer-by-layer assembly: macroscale anti-inflammatory conductive coatings with optoelectronic tunability, Biomacromolecules 18 (6) (2017) 1908-1917.

[27] F. Solano, M.elanins: skin pigments and much more-types, structural models, biological functions, and formation routes, New J. Sci. (2014) 1-28 2014.

[28] Company, the good scents. 2019. tgsc information system http://www. thegoodscentscompany.com/data/rw1359121.html (Accessed 6 December 2019).

[29] M.M. Rageh, R.H. EL-Gebaly, H. Abou-Shady, D.G. Amin, Melanin nanoparticles (MNPs) provide protection against whole-body V-Irradiation in mice via restoration of hematopoietic tissues, Mol. Cell. Biochem. 399 (2015) 59-69 1-2.

[30] A.D. Schweitzer, E. Revskaya, P. Chu, V. Pazo, M. Friedman, J.D. Nosanchuk, S. Cahill, S. Frases, A. Casadevall, E. Dadachova, Melanin-Covered nanoparticles for protection of bone marrow during radiation therapy of cancer, Int. J. Radiat. Oncol. 78 (5) (2010) 1494-1502.

[31] M. Chu, W. Hai, Z. Zhang, F. Wo, Q. Wu, Z. Zhang, Y. Shao, D. Zhang, L. Jin D. Shi, Melanin nanoparticles derived from a homology of medicine and food for sentinel lymph node mapping and photothermal in vivo cancer therapy, Biomaterials 91 (2016) 182-199.

[32] Q. Jiang, Z. Luo, Y. Men, P. Yang, H. Peng, R. Guo, Y. Tian, Z. Pang, W. Yang, Red blood cell membrane-camouflaged melanin nanoparticles for enhanced photothermal therapy, Biomaterials 143 (2017) 29-45.

[33] J. Wang, H. Liu, Y. Liu, C. Chu, Y. Yang, Y. Zeng, W. Zhang, G. Liu, Eumelanin-Fe304 hybrid nanoparticles for enhanced $\mathrm{mr} / \mathrm{pa}$ imaging-assisted local photothermolysis, Biomater. Sci. 6 (3) (2018) 586-595.

[34] G. Perna, G. Palazzo, A. Mallardi, V. Capozzi, Fluorescence properties of natural eumelanin biopolymer, J. Lumin. 131 (8) (2011) 1584-1588.

[35] H. Ou-Yang, G. Stamatas, N. Kollias, Spectral responses of melanin to ultraviolet a irradiation, J. Invest. Dermatol. 122 (2) (2004) 492-496.

[36] M. Brenner, V.J. Hearing, The protective role of melanin against uv damage in human skin, Photochem. Photobiol. 84 (3) (2008) 539-549.

[37] D.A. Novikov, V.P. Kurchenko, I.I. Azarko, [Photoprotective properties of melanins from grape (Vitis vinifera) and black tea (Thea sinensis)], Radiatsionnaia Biol. Radioecol. 41 (6) (2001) 664-670.

[38] A. Pezzella, L. Capelli, A. Costantini, G. Luciani, F. Tescione, B. Silvestri, G. Vitiello, F. Branda, Towards the development of a novel bioinspired functional material: synthesis and characterization of hybrid tio2/dhica-melanin nanoparticles, Mater. Sci. Eng. C 33 (1) (2013) 347-355.
[39] P. Srisuk, V.M. Correlo, I.B. Leonor, P. Palladino, R.L. Reis, Redox activity of melanin from the ink sac of sepia officinalis by means of colorimetric oxidative assay, Nat. Prod. Res. 30 (8) (2016) 982-986.

[40] da Silva, L. P., S. Oliveira, R.P. Pirraco, T.C. Santos, R.L. Reis, A.P. Marques, V.M. Correlo, Eumelanin-Releasing spongy-like hydrogels for skin re-epithelialization purposes, Biomed. Mater. 12 (2) (2017)

[41] L. Zecca, C. Bellei, P. Costi, A. Albertini, E. Monzani, L. Casella, M. Gallorini, L. Bergamaschi, A. Moscatelli, N.J. Turro, et al. new melanic pigments in the human brain that accumulate in aging and block environmental toxic metals, Proc. Natl. Acad. Sci. U. S. A. 105 (45) (2008) 17567-17572.

[42] A.G.-E. Costin, Is there an answer? IUBMB Life (International Union Biochem. Mol. Biol. Life) 56 (1) (2004) 47-49.

[43] K. Stepień, A. Dzierzega-Lecznar, I. Tam, The role of neuromelanin in parkinson's disease-new concepts, Wiad. Lek. 60 (2007) 563-569 11-12.

[44] A. Büngeler, B. Hämisch, O. Strube, A. Büngeler, B. Hämisch, O.I. Strube, The supramolecular buildup of eumelanin: structures, mechanisms, controllability, Int. J. Mol. Sci. 18 (9) (2017) 1901.

[45] H. Lee, J. Rho, P.B. Messersmith, Facile conjugation of biomolecules onto surfaces via mussel adhesive protein inspired coatings, Adv. Mater. 21 (4) (2009) $431-434$.

[46] M. Bridelli, R. Capelletti, P.R. Crippa, Electret state and hydrated structure of melanin, J. Electroanal. Chem. Interfacial Electrochem. 128 (1981) 555-567.

[47] C. Sarzanini, E. Mentasti, O. Abollino, M. Fasano, S. Aime, Metal ion content in sepia officinalis melanin, Mar. Chem. 39 (4) (1992) 243-250.

[48] Y. Liu, L. Hong, V.R. Kempf, K. Wakamatsu, S. Ito, J.D. Simon, Ion-Exchange and adsorption of fe(iii) by sepia melanin, Pigment Cell Res 17 (3) (2004) 262-269.

49] L. Hong, J.D. Simon, Current understanding of the binding sites, capacity, affinity, and biological significance of metals in melanin. J. Phys. Chem. B 111 (28) (2007) 7938-7947.

[50] J.D. Simon, L. Hong, D.N. Peles, Insights into melanosomes and melanin from some interesting spatial and temporal properties, J. Phys. Chem. B 112 (42) (2008) 13201-13217.

[51] K.-Y. Ju, Y. Lee, S. Lee, S.B. Park, J.-K. Lee, Bioinspired polymerization of dopamine to generate melanin-like nanoparticles having an excellent free-radical-scavenging property, Biomacromolecules 12 (3) (2011) 625-632.

[52] B. Silvestri, G. Vitiello, G. Luciani, V. Calcagno, A. Costantini, M. Gallo, S. Parisi, S. Paladino, M. Iacomino, G. D'Errico, et al. probing the eumelanin-silica interface in chemically engineered bulk hybrid nanoparticles for targeted subcellular antioxidant protection, ACS Appl. Mater. Interfaces 9 (43) (2017) 37615-37622.

53] Y. Gao, X. Wu, L. Zhou, Y. Su, C.-M. Dong, A sweet polydopamine nanoplatform for synergistic combination of targeted chemo-photothermal therapy, Macromol. Rapid Commun. 36 (10) (2015) 916-922

[54] X. Wang, J. Zhang, Y. Wang, C. Wang, J. Xiao, Q. Zhang, Y. Cheng, Multi-Responsive photothermal-chemotherapy with drug-loaded melanin-like nanoparticles for synergetic tumor ablation, Biomaterials 81 (2016) 114 124.

[55] Y. Liu, K. Ai, J. Liu, M. Deng, Y. He, L. Lu, Dopamine-Melanin colloida nanospheres: an efficient near-infrared photothermal therapeutic agent for in vivo cancer therapy, Adv. Mater. 25 (9) (2013) 1353-1359.

[56] Q. Fan, K. Cheng, X. Hu, X. Ma, R. Zhang, M. Yang, X. Lu, L. Xing, W. Huang, S.S. Gambhir, et al., Transferring biomarker into molecular probe: melanin nanoparticle as a naturally active platform for multimodality imaging, J. Am. Chem. Soc. 136 (43) (2014) 15185-15194.

[57] D. Hu, C. Liu, L. Song, H. Cui, G. Gao, P. Liu, Z. Sheng, L. Cai, Indocyanine green-loaded polydopamine-iron ions coordination nanoparticles for photoacoustic/magnetic resonance dual-modal imaging-guided cancer photothermal therapy, Nanoscale 8 (39) (2016) 17150-17158.

[58] Y. Li, C. Jiang, D. Zhang, Y. Wang, X. Ren, K. Ai, X. Chen, L. Lu, Targeted polydopamine nanoparticles enable photoacoustic imaging guided chemo-photothermal synergistic therapy of tumor, Acta Biomater 47 (2017) 124-134.

[59] S. Cho, W. Park, D.-H. Kim, Silica-Coated metal chelating-melanin nanoparticles as a dual-modal contrast enhancement imaging and therapeutic agent, ACS Appl. Mater. Interfaces 9 (1) (2017) 101-111.

[60] R. Zhang, Q. Fan, M. Yang, K. Cheng, X. Lu, L. Zhang, W. Huang, Z. Cheng, Engineering melanin nanoparticles as an efficient drug-delivery system for imaging-guided chemotherapy, Adv. Mater. 27 (34) (2015) 5063-5069.

[61] F. Scognamiglio, A. Travan, G. Turco, M. Borgogna, E. Marsich, M. Pasqua S. Paoletti, I. Donati, Adhesive coatings based on melanin-like nanoparticles for surgical membranes, Colloids Surfaces B Biointerfaces 155 (2017) 553-559.

[62] M. Sheliakina, A.B. Mostert, P. Meredith, An all-solid-state biocompatible ion-to-electron transducer for bioelectronics, Mater. Horizons 5 (2) (2018) 256-263.

[63] M.M. Rageh, R.H. El-Gebaly, Melanin nanoparticles: antioxidant activities and effects on $\gamma$-Ray-Induced dna damage in the mouse, Mutat. Res. Toxicol. Environ. Mutagen. 828 (2018) 15-22.

[64] N. Belemets, N. Kobyliak, O. Virchenko, T. Falalyeyeva, T. Olena, P. Bodnar, O. Savchuk, T. Galenova, M. Caprnda, L.;. Rodrigo, et al., Effects of polyphenol compounds melanin on nafld/nash prevention, Biomed. Pharmacother. 88 (2017) 267-276

[65] M. Araujo, R. Viveiros, T.R. Correia, I.J. Correia, V.D. Bonifacio, T. Casimiro, A. Aguiar-Ricardo, A. Natural Melanin, Potential pH-Responsive drug release device, Int. J. Pharm. 469 (1) (2014) 140-145.

[66] A.-K. Rimpelä, M. Reinisalo, L. Hellinen, E. Grazhdankin, H. Kidron, E.M. de Amo, Implications of melanin binding in ocular drug delivery, Adv. Drug Deliv. Rev. 126 (2018) 23-43 
[67] R. Batul, T. Tamanna, A. Khaliq, A. Yu, Recent progress in the biomedical applications of polydopamine nanostructures, Biomater. Sci. 5 (7) (2017) 1204-1229.

[68] L. Cheng, C. Wang, L. Feng, K. Yang, Z. Liu, Functional nanomaterials for phototherapies of cancer, Chem. Rev. 114 (21) (2014) 10869-10939.

[69] R.W.Y. Habash, R. Bansal, D. Krewski, H.T. Alhafid, Thermal therapy, part 1: an introduction to thermal therapy, Crit. Rev. Biomed. Eng. 34 (6) (2006) 459-489.

[70] S. Luo, E. Zhang, Y. Su, T. Cheng, C. Shi, A review of nir dyes in cancer targeting and imaging, Biomaterials 32 (29) (2011) 7127-7138.

[71] X. Wang, J. Sheng, M. Yang, Melanin-Based nanoparticles in biomedical applications: from molecular imaging to treatment of diseases, Chinese Chem. Lett. (2018).

[72] J. Xia, J. Yao, L.V. Wang, Photoacoustic tomography: principles and advances, Electromagn. Waves (Cambridge, Mass.) 147 (2014) 1-22.

[73] K.-Y. Ju, J. Kang, J. Pyo, J. Lim, J.H. Chang, J.-K. Lee, pH-Induced aggregated melanin nanoparticles for photoacoustic signal amplification, Nanoscale 8 (30) (2016) 14448-14456.

[74] F.ud Din, W. Aman, I. Ullah, O.S. Qureshi, O. Mustapha, S. Shafique, A. Zeb, Effective use of nanocarriers as drug delivery systems for the treatment of selected tumors, Int. J. Nanomedicine 12 (2017) 7291-7309 Volume.
[75] D.M. Connor, Gold nanoparticles for the delivery of cancer therapeutics, in: In Advances in Cancer Research, Vol. 139, Academic Press, 2018, pp. 163-184.

[76] J. Li, Z. Zhuang, B. Jiang, P. Zhao, C. Lin, C. Lin, Advances and perspectives in nanoprobes for noninvasive lymph node mapping, Nanomedicine 10 (6) (2015) 1019-1036.

[77] C.-M.J. Hu, L. Zhang, S. Aryal, C. Cheung, R.H. Fang, L. Zhang, Erythrocyte membrane-camouflaged polymeric nanoparticles as a biomimetic delivery platform, Proc. Natl. Acad. Sci. U. S. A. 108 (27) (2011) 10980-10985.

[78] F. Mazuel, A. Espinosa, N. Luciani, M. Reffay, R. Le Borgne, L. Motte, K. Desboeufs, A Michel, T. Pellegrino, Y. Lalatonne, et al massive intracellular biodegradation of iron oxide nanoparticles evidenced magnetically at single-endosome and tissue levels, ACS Nano 10 (8) (2016) 7627-7638.

[79] E. Vahidzadeh, A.P. Kalra, K. Shankar, Melanin-Based electronics: from proton conductors to photovoltaics and beyond, Biosens. Bioelectron. 122 (2018) 127-139.

[80] A. Chassepot, V. Ball, Human serum albumin and other proteins as templating agents for the synthesis of nanosized dopamine-eumelanin, J. Colloid Interface Sci. 414 (2014) 97-102. 